University of Tennessee Health Science Center

UTHSC Digital Commons

$12-2019$

\title{
Validation Testing of a Synthetic Spine and Upgraded Protocol for a Biomechanical Evaluation of a Lumbar Spinal Orthosis
}

Daniel Hoyer

University of Tennessee Health Science Center

Follow this and additional works at: https://dc.uthsc.edu/dissertations

Part of the Analytical, Diagnostic and Therapeutic Techniques and Equipment Commons

\section{Recommended Citation}

Hoyer, Daniel (https://orcid.org/0000-0002-9369-9038), "Validation Testing of a Synthetic Spine and Upgraded Protocol for a Biomechanical Evaluation of a Lumbar Spinal Orthosis" (2019). Theses and Dissertations (ETD). Paper 505. http://dx.doi.org/10.21007/etd.cghs.2019.0566.

This Thesis is brought to you for free and open access by the College of Graduate Health Sciences at UTHSC Digital Commons. It has been accepted for inclusion in Theses and Dissertations (ETD) by an authorized administrator of UTHSC Digital Commons. For more information, please contact jwelch30@uthsc.edu. 


\title{
Validation Testing of a Synthetic Spine and Upgraded Protocol for a Biomechanical Evaluation of a Lumbar Spinal Orthosis
}

\begin{abstract}
Low back pain (LBP) is highly prevalent in all walks of life. Standard conservative treatment methods may work for some, but others go on to have spinal injections, opioids, or surgical procedures to alleviate pain. The Distractive and Mobility-Enabling Orthosis (DMO) was developed to meet the need for a conservative, drug-free treatment method. In this research, the low back support test protocol for evaluating DMO success was upgraded with a new synthetic lumbar spine model. The latest generation of the DMO project was then evaluated using the new system in a laboratory setting and on a pilot physical therapy patient.First, a biomechanical evaluation study of the new full-length synthetic lumbar spine model was performed to validate its use in the low back support test protocol. Markers placed at each vertebral body level enabled the local and global instantaneous axis of rotation of each spinal segment to be defined. Combined with moment data, the moment-rotational stiffness properties was compared to similar published data from other in vitro and in vivo studies of the lumbar spine. These comparisons provided validation and justification for using the synthetic model in the low back support test protocol. The design of the fourth generation (DMO4) is then detailed. DMO4 borrowed some characteristics from previous generations but was modified to lower the complexity of the parts and increase user comfort. DMO4 utilized hip and torso belts to secure itself to the patient. A distractive force applied via gas springs on the lateral sides of the belts separated the belts in order to offload the lumbar spine. DMO4 reduced the side profile, reduced the component count by half, and redesigned the torso belt to be more conforming to patient anatomy.DMO4 was then evaluated with the low back support test protocol that was set to match daily living activities (DLAs) and offload an average human torso weight. Placed under a $150 \mathrm{~N}$ vertical load, DMO4 successfully offloaded the lumbar spine in upright stance through $25^{\circ}$ of flexion and $10^{\circ}$ of extension. DMO4 was also successful at providing unconstrained axial rotation beyond the range required for DLAs. Pilot data of a physical therapy patient with low back pain was ascertained. The patient wore the DMO during six physical therapy sessions over a four week period. At each session, the patient's pain score significantly dropped to a pain rating score of one and the modified Oswestry Disability Index also saw a reduction.
\end{abstract}

\section{Document Type}

Thesis

\section{Degree Name}

Master of Science (MS)

\section{Program}

Biomedical Engineering

Research Advisor

Denis J. DiAngelo, Ph.D.

\section{Keywords}

Low Back Pain, Orthosis, Synthetic Spine

\section{Subject Categories}

Analytical, Diagnostic and Therapeutic Techniques and Equipment | Medicine and Health Sciences 


\section{Validation Testing of a Synthetic Spine and Upgraded Protocol for a Biomechanical Evaluation of a Lumbar Spinal Orthosis}

Author:

Daniel Hoyer
Advisor:

Denis J. DiAngelo, Ph.D.

A Thesis Presented for The Graduate Studies Council of

The University of Tennessee Health Science Center

in Partial Fulfillment of the Requirements for the

Master of Science degree from

The University of Tennessee

in

The Joint Graduate Program in Biomedical Engineering: Biomechanics College of Graduate Health Sciences 
Copyright @ 2019 by Daniel Hoyer. All rights reserved. 


\section{DEDICATION}

This work is dedicated to my family, Jessica Hoyer, Keith and Cheryl Hoyer, and Mark and Candy Maddox. Without their support this would not have been possible. 


\section{ACKNOWLEDGEMENTS}

I would like to thank everyone who had a part in my educational experience at the University of Tennessee Health Science Center. First, I'd like to thank my advisor, Dr. DiAngelo, who provided an opportunity for me to join his team in the BioRobotics Laboratory. It had been a long term goal of mine to enter the biomedical field and it would not have happened without his support. The wide range of experiences I received during my time in lab is unlike any other and they afforded me the means to take my career to the next level. I would also like to thank the members of my committee, Dr. Kasser and Dr. Mihalko, for their input and guidance throughout this process. Chloe Chung deserves a thank you for all the direction she gave me starting from day one and for her valuable input within my projects. Finally, I would like to thank my wife Jessica for pushing me to go back to school and empowering me to pursue my degree. 


\begin{abstract}
Low back pain (LBP) is highly prevalent in all walks of life. Standard conservative treatment methods may work for some, but others go on to have spinal injections, opioids, or surgical procedures to alleviate pain. The Distractive and MobilityEnabling Orthosis (DMO) was developed to meet the need for a conservative, drug-free treatment method. In this research, the low back support test protocol for evaluating DMO success was upgraded with a new synthetic lumbar spine model. The latest generation of the DMO project was then evaluated using the new system in a laboratory setting and on a pilot physical therapy patient.
\end{abstract}

First, a biomechanical evaluation study of the new full-length synthetic lumbar spine model was performed to validate its use in the low back support test protocol. Markers placed at each vertebral body level enabled the local and global instantaneous axis of rotation of each spinal segment to be defined. Combined with moment data, the moment-rotational stiffness properties was compared to similar published data from other in vitro and in vivo studies of the lumbar spine. These comparisons provided validation and justification for using the synthetic model in the low back support test protocol.

The design of the fourth generation (DMO4) is then detailed. DMO4 borrowed some characteristics from previous generations but was modified to lower the complexity of the parts and increase user comfort. DMO4 utilized hip and torso belts to secure itself to the patient. A distractive force applied via gas springs on the lateral sides of the belts separated the belts in order to offload the lumbar spine. DMO4 reduced the side profile, reduced the component count by half, and redesigned the torso belt to be more conforming to patient anatomy.

DMO4 was then evaluated with the low back support test protocol that was set to match daily living activities (DLAs) and offload an average human torso weight. Placed under a $150 \mathrm{~N}$ vertical load, DMO4 successfully offloaded the lumbar spine in upright stance through $25^{\circ}$ of flexion and $10^{\circ}$ of extension. DMO4 was also successful at providing unconstrained axial rotation beyond the range required for DLAs.

Pilot data of a physical therapy patient with low back pain was ascertained. The patient wore the DMO during six physical therapy sessions over a four week period. At each session, the patient's pain score significantly dropped to a pain rating score of one and the modified Oswestry Disability Index also saw a reduction. 


\section{TABLE OF CONTENTS}

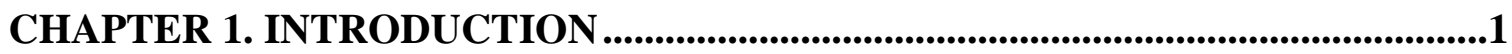

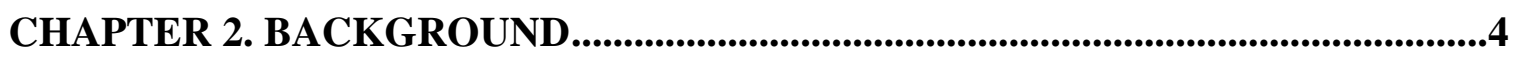

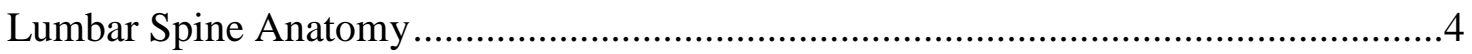

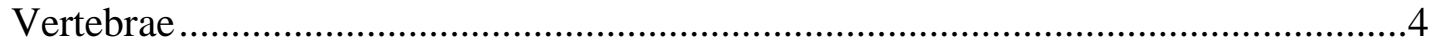

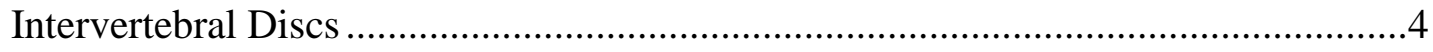

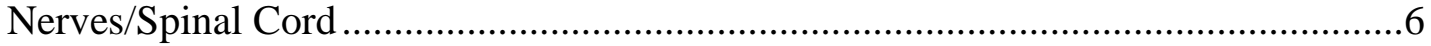

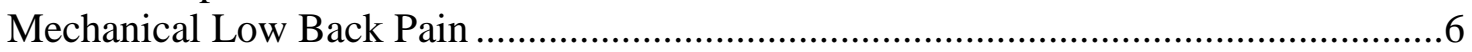

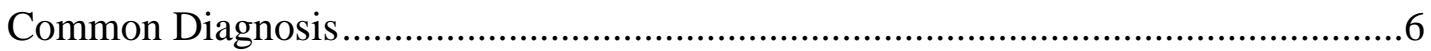

Current Conservative Treatment Options ……………...........................................

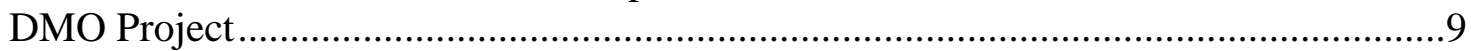

Design of the Previous DMOs .............................................................................

Design of the Testing Setup, Spine, and LARS ....................................................11

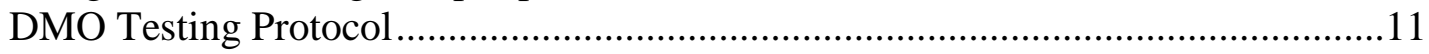

\section{CHAPTER 3. BIOMECHANICAL EVALUATION OF A FULL-LENGTH} (T12-S) SYNTHETIC LUMBAR SPINE MODEL ...........................................................14

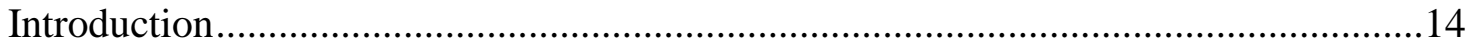

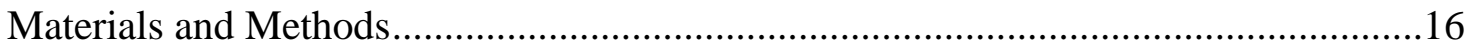

Synthetic Lumbar Spine Model and Testing Platform.............................................16

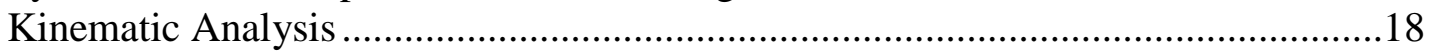

Method for Analyzing Multi-Level Spine Model Segmentally …….........................18

Testing Protocol ..........................................................................................21

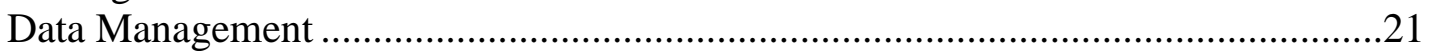

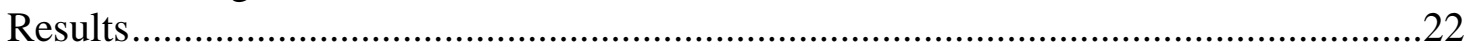

Global Stiffness and MSU Rotational Contribution ................................................22

Global CoR and IAR Patterns .........................................................................22

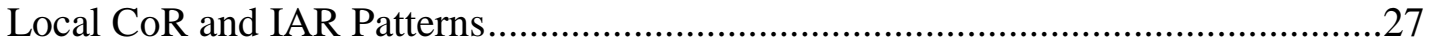

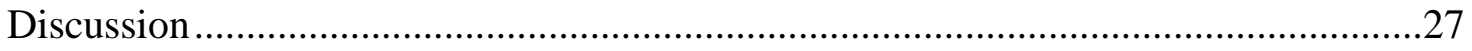

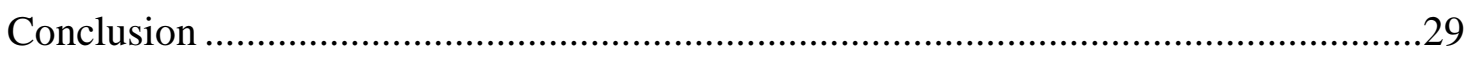

CHAPTER 4. DESIGN OF DMO4 .............................................................................31

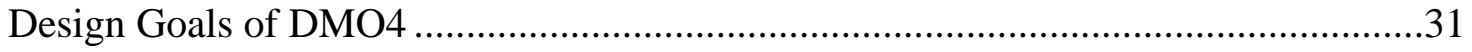

DMO4 Body Engagement …………..................................................................

DMO4 Distractive Force Mechanism.........................................................................33

DMO4 Mobility-Enabling Component .......................................................................33

CHAPTER 5. A BIOMECHANICAL EVALUATION OF AN IMPROVED UNLOADING AND MOBILITY-ENABLING LUMBAR SPINAL ORTHOSIS ....35

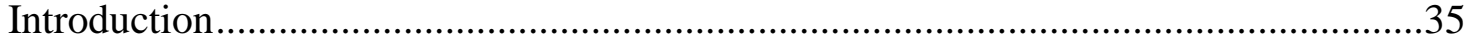

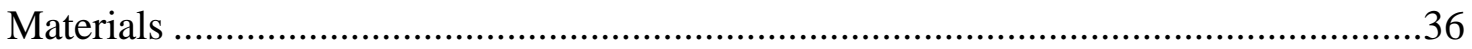

Distractive and Mobility-Enabling Orthosis ...............................................................36 


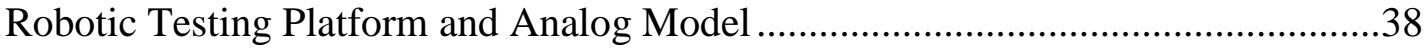

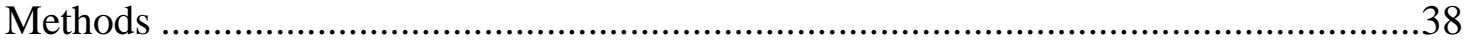

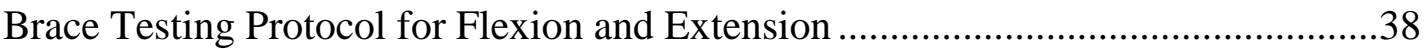

Brace Testing Protocol for Axial Rotation .......................................................4

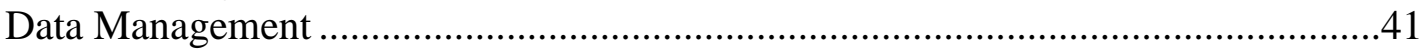

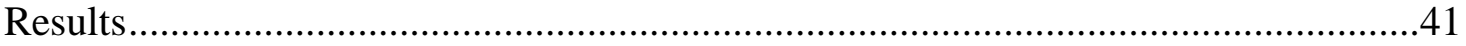

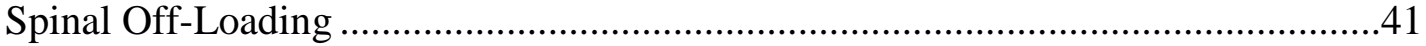

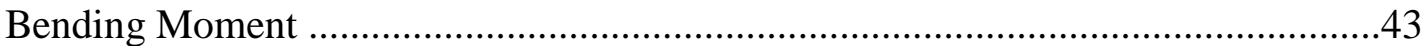

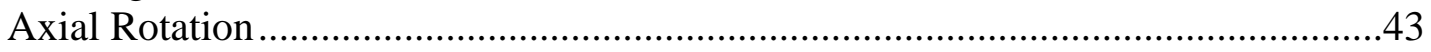

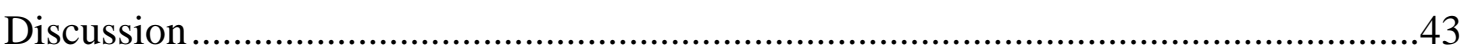

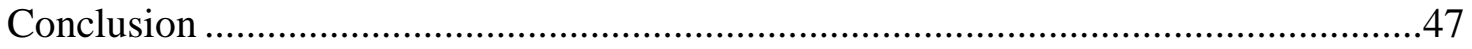

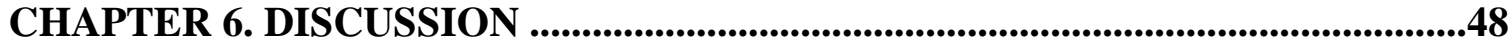

Additional Synthetic Spine Tests - Eccentric Load Condition ....................................48

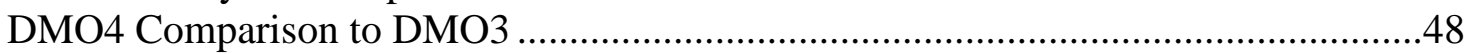

Design Changes Between DMO3 and DMO4 ….............................................48

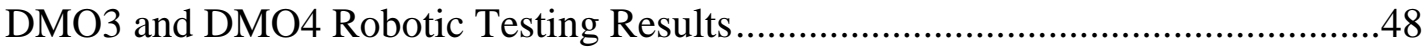

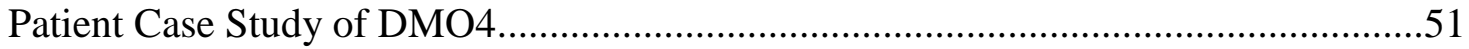

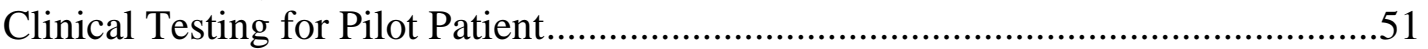

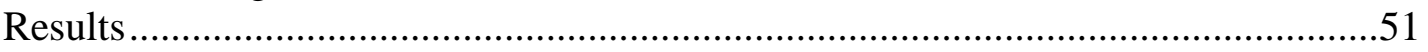

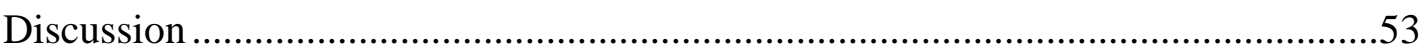

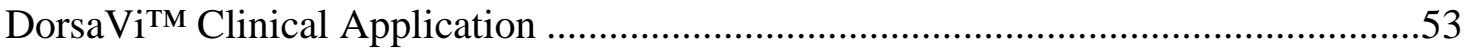

CHAPTER 7. CONCLUSION

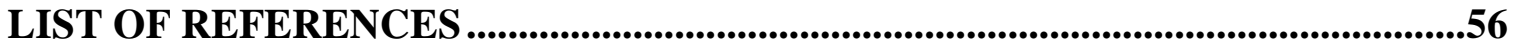

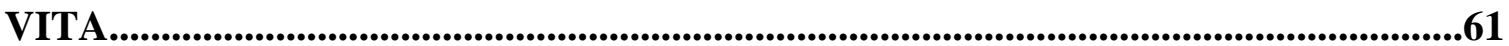




\section{LIST OF TABLES}

Table 3-1: Flexion and extension response per motion segment unit. ........................23

Table 5-1: DMO4 compressive load values at upright stance and at end ranges of

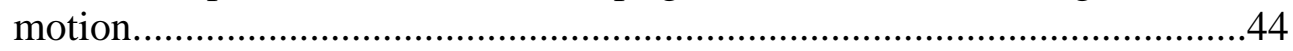

Table 5-2: DMO4 bending moment values at end ranges of motion. .........................45

Table 5-3: DMO4's unconstrained $(<0.1 \mathrm{Nm}$ of resistance) axial rotation degree

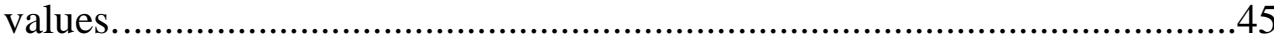




\section{LIST OF FIGURES}

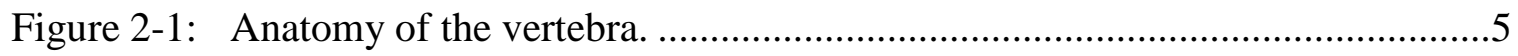

Figure 2-2: Conservative LSO treatment options for LBP ......................................

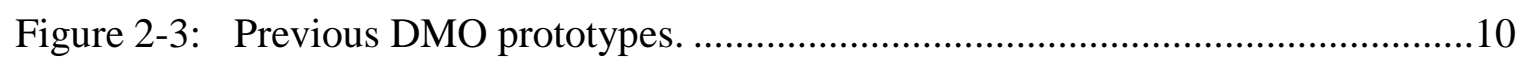

Figure 2-4: RTP with human analog model and DMO installed.................................12

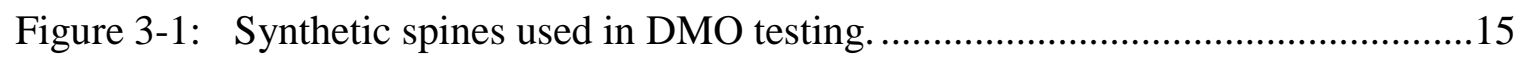

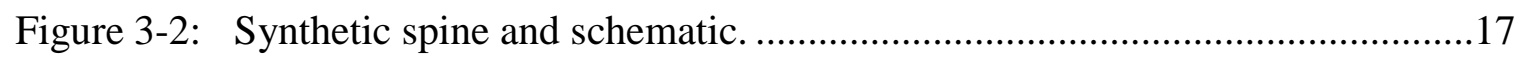

Figure 3-3: Experimental set-up of a synthetic lumbar spine mounted in the robotic testing platform.

Figure 3-4: Method for determining the local IAR of upper body (UB) relative to the

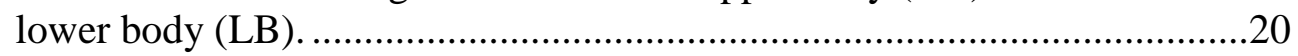

Figure 3-5: Comparison of moment-rotation stiffness properties. .............................23

Figure 3-6: Comparison of percent global segmental motion response between synthetic model and in vitro pure moment test results.

Figure 3-7: Comparison of percent global segmental motion response between synthetic model and in vivo motion patterns. .25

Figure 3-8: Global IAR patterns of each spinal level. ..............................................26

Figure 3-9: Local CoR and IAR patterns of each motion segment unit. .......................28

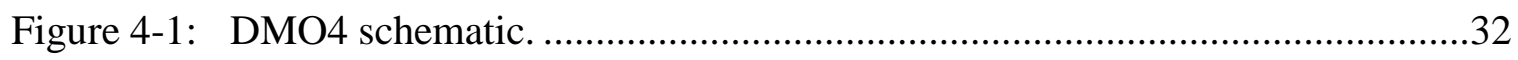

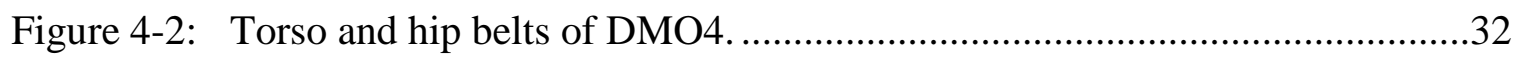

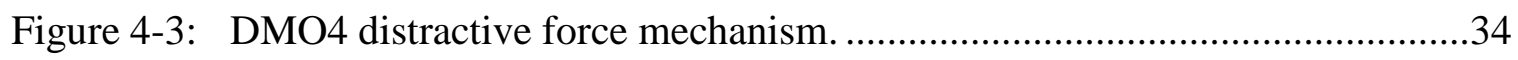

Figure 5-1: Front and side view of DMO4 displaying key components. .......................37

Figure 5-2: RTP and the human analog model with and without the pelvic girdle.........39

Figure 5-3: DMO4 installed onto the analog model for flexion and extension testing. ..40

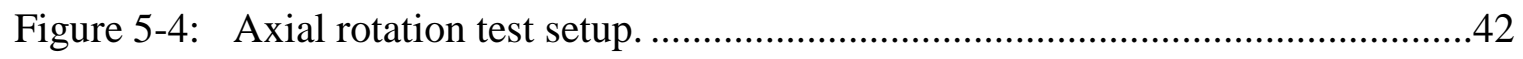

Figure 5-5: Spinal offloading of DMO4 in upright stance and throughout extended ranges of motion. 
Figure 5-6: Resistance to bending of DMO4 in upright stance and throughout extended ranges of motion.

Figure 5-7: Brace offloading capacity or brace load comparison between the latest models.

Figure 5-8: Resistance to bending or brace effect comparison between the latest models.

Figure 6-1: Eccentric load test setup

Figure 6-2: Global IAR found from EL protocol and initial rotation for PM protocol...49

Figure 6-3: Profile view comparison of DMO3 and DMO4. .50

Figure 6-4: NPRS scores for patient 1 before and after wearing DMO4 during PT visits.

Figure 6-5: mODI scores for patient 1.

Figure 6-6: DorsaVi ${ }^{\mathrm{TM}}$ low back module flexion results of a healthy patient wearing DMO4. 


\section{LIST OF ABBREVIATIONS}

\begin{tabular}{|c|c|}
\hline $\mathrm{AF}$ & Annulus Fibrosis \\
\hline CCD & Charged Coupled Device \\
\hline CoR & Center of Rotation \\
\hline DDD & Degenerative Disc Disease \\
\hline DFM & Distractive Force Mechanism \\
\hline DLA & Daily Living Activity \\
\hline DMO & Distractive and Mobility-enabling Orthosis \\
\hline EL & Eccentric Load \\
\hline IAR & Instantaneous Axis of Rotation \\
\hline IVD & Intervertebral Disc \\
\hline LB & Lower Body \\
\hline LBP & Low Back Pain \\
\hline LSCS & Lumbar Spine Core Strengthening \\
\hline LSO & Lumbar Sacral Orthosis \\
\hline mODI & Modified Oswestry Disability Index \\
\hline MSU & Motion Segment Unit \\
\hline NP & Nucleus Pulposus \\
\hline NPRS & Numerical Pain Rating Scale \\
\hline $\mathrm{PM}$ & Pure Moment \\
\hline ROM & Range of Motion \\
\hline RTP & Robotic Testing Platform \\
\hline UB & Upper Body \\
\hline
\end{tabular}




\section{CHAPTER 1. INTRODUCTION}

Low back pain (LBP) is a prevalent problem by all measures - it affects $30-45 \%$ of the population at any given time [1-5], is one of the top 5 reasons for visits to a healthcare professional $[6,7]$, and is the number one cause of disability for patients under the age of 45 years $[1,8,9]$. A majority of this pain is mechanical in nature [10-13], and initial treatment attempts begin with conservative methods [11,12]. This conservative treatment usually begins with over the counter medication followed by many different forms of physical therapy strengthening [7, 14], water therapy, distraction tables, passive therapy (heat packs, ice, TENS), and exercise. When conservative methods do not work, more serious or invasive options such as opioids, spinal injections, or surgery are used to treat LBP.

Unfortunately for some people, pain may be a limiting factor in the success of standard conservative methods. This limited success and the ease of access to narcotics has led to an increase of opioid prescriptions $[6,15,16]$. With the rampant rise of opioid addiction and limitations with many current conservative treatment methods, there remains a need for an alternative conservative approach $[6,17,18]$.

This motivation led to the development of a dynamic Lumbar Sacral Orthosis (LSO) that was designed to apply a distractive load to the lumbar spine while allowing unconstrained movement. This combination of features should decompress the spine and alleviate pain - offering healthcare providers a solution to treat LBP to lessen the occurrence of prescribing addictive opioid medications. The brace functions similarly to a traction table in that it alleviates pain by applying distraction to the spine. Unlike a traction table, however, this orthosis allows for local motion of the lumbar spine in all planes of movement (sagittal, transverse, and coronal) with minimal additional muscle recruitment. The overarching goal of the dynamic LSO was to enable patients to perform standard physical therapy exercises and various daily living activities (DLAs) while receiving benefits of traction therapy.

In order to test the Distractive and Mobility-Enabling Orthosis (DMO) prototypes and similar LSO braces in a controlled laboratory setting, a human analog model was developed. This model linked two six axis load cells within a robot testing platform [19] by utilizing a reinforced mannequin torso and a custom created biomimetic lumbar spine developed from cadaver anatomy [20]. The analog model was manipulated to simulate DLAs by flexing through different ranges of motion (ROMs). Forces and moments were read at each of the load cells and were analyzed to determine the brace's response during each of the actions.

Unfortunately, after years of testing, the biomimetic spine utilized in the testing protocol began to show wear and needed to be replaced before the next iteration of the DMO project could be fully developed. A full length (T12-S) synthetic biomechanical lumbar spine was sourced from Sawbones Inc., Pacific Research Laboratories (Sawbones 
\#3430). This lumbar spine model needed to be validated prior to being added to the existing test protocol to enable evaluation of DMO4.

The new synthetic spine model was evaluated on the robotic testing platform (RTP) under pure moment (PM) loading conditions. Charged coupled device (CCD) cameras and near infrared trackers were used to measure the motion of each vertebral body. A new method to determine the kinematic properties of the lumbar spine was developed to evaluate the synthetic lumbar spine at the segmental level. With the replacement spine meeting validation requirements to previous cadaveric models and similar synthetic specimens, the synthetic spine was integrated into the test platform for DMO evaluation.

The previous version of the DMO project, DMO3, met the design goal of successfully offloading $150 \mathrm{~N}$ through $25^{\circ}$ of flexion, $10^{\circ}$ of extension, and allowed for $10^{\circ}$ of axial rotation [21]. This $150 \mathrm{~N}$ target load enabled the brace to offload half of a 750N person's upper torso weight [22] through a sagittal plane range suitable for most DLAs [23]. However, DMO3 was not without limitations. DMO3 was bulky to wear and difficult to put on, was limited to a single offloading force, and was difficult to fabricate and repair. A new iteration, DMO4, was created to improve wear ability, simplify the design, and provide configurable options for patients.

The objectives of the following work were:

1) To validate the synthetic T12S1 spine's capacity and resistance to bending under simulated flexion and extension, determine the local and global instantaneous axis of rotation (IAR) for each segment, and incorporate the validated spine into the DMO testing protocol.

2) To improve upon the design of DMO3: Reduce complexity \& improve patient wearability while maintaining offloading capacity.

3) To determine DMO4's spinal offloading capabilities and resistance to bending under simulated flexion, extension, and axial rotation.

A description of the content of each subsequent chapter of this thesis is provided below.

Chapter 2 provides a description of spinal anatomy, clinical problems that attribute to LBP, and the current non-surgical methods for treating it. A brief overview of the earlier designs of the dynamic back orthoses is given, as well as the testing protocol used to quantify the biomechanical properties of past braces designs and the testing methods used to assess them.

Chapter 3 is a copy of a manuscript published entitled "Biomechanical Evaluation of a Full-length (T12-S) Synthetic Lumbar Spine Model" [24]. The paper covers the work done to evaluate a new full length synthetic lumbar spine model. The synthetic model 
will be added to the existing testing protocol and be used to study the new dynamic spinal orthosis. Included in this manuscript is the presentation of a novel method for determining the IAR of individual spinal motion segment units.

Chapter 4 describes the upgraded design changes made to the dynamic back support brace in DMO4.

Chapter 5 is second manuscript that resulted from the work completed in this thesis entitled "A Biomechanical Evaluation of an Improved Unloading and MobilityEnabling Lumbar Spinal Orthosis". This paper summarizes the work done using the new full length synthetic lumbar spine model and modified testing protocol to determine the biomechanical properties of DMO4.

Chapter 6 highlights the findings from a single case study of a LBP patient wearing DMO4 as per an approved IRB protocol (\#16-04546-FB). Also included are additional findings from the experimental testing of the synthetic spine model as well as a potential clinical measure of the DMO performance.

Chapter 7 summarizes the collective findings from the completed work. 


\section{CHAPTER 2. BACKGROUND}

\section{Lumbar Spine Anatomy}

The lumbar spine is made up of a column of five vertebrae and intervertebral discs (IVDs) starting below the ribs and ending at the sacrum. This section of spine is where most weight bearing and body movement takes place [25]. Due to the high loads and repetitive motion, many individuals can experience unrelenting pain stemming from the lumbar spine during DLAs. Basic anatomy for a typical vertebrae pair is shown in Figure 2-1.

\section{Vertebrae}

Vertebrae provide structure and rigidity to the lumbar spine and consist of several different components. The largest component is the vertebral body; this is a portion of round bone that carries vertical loads. Protecting the spinal cord are the pedicle and lamina that extend out from the dorsal side of the vertebral body. The transverse and spinous process, stemming off the lamina laterally and posteriorly, provide anchor points for the ligaments and muscles that aid in stabilization and motion. Lastly, facet joints, or articular processes, link the adjacent vertebrae together. In the lumbar spine, these facet joints allow for motion in the sagittal plane, but largely restrict axial rotation and coronal plane motion [26].

\section{Intervertebral Discs}

IVDs are stacked in between the vertebrae cushioning vertical loads. Paired with the linked vertebral bodies, lamina, and facet joints, IVDs allow room for nerve roots to pass from the spinal column out to the body. With this task in mind, IVDs must be stiff enough to prevent collapsing and nerve impingement, yet flexible enough for lumbar motion to occur. To handle this demand, IVDs are composed of two components, the annulus fibrosis (AF) and nucleus pulposus (NP), sandwiched between cartilaginous vertebral endplates. The AF consists of collagen fibers wrapped concentrically around the $\mathrm{NP}$ and are arranged so that they may withstand the cylindrical forces within the disc. The NP is a hydrated gel of proteoglycans nested within the AF. This gel prevents internal collapse of the AF and acts to distribute forces. With no direct blood supply, the IVD largely receives nutrients through diffusion. Therefore movement of the lumbar spine greatly aids the nutrition of the disc by forcing water and nutrients into and out of the disc space [26]. 


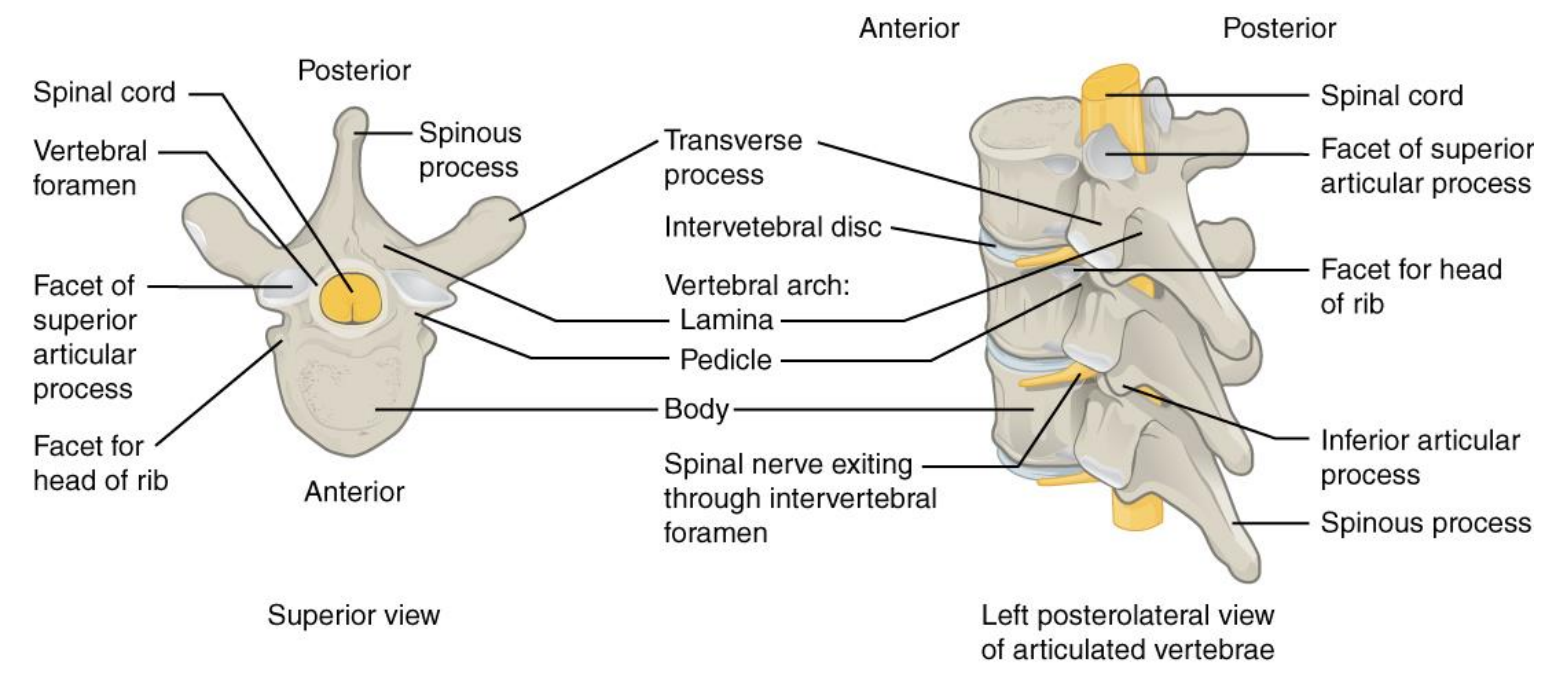

Figure 2-1: Anatomy of the vertebra.

Source: Reprinted with permission. J. G. Betts, K. A. Young, J. A. Wise, E. Johnson, B. Poe, D. H. Kruse, et al., Anatomy \& Physiology., pp. 264: OpenStax 2013, https://openstax.org/books/anatomy-and-physiology/pages/7-3-the-vertebral-column [27]. 


\section{Nerves/Spinal Cord}

The spinal cord lays within the lamina of vertebrae connecting the brain to the rest of the body. Within the intervertebral foramen, spinal nerves branch off the spinal cord by dorsal and ventral roots. Should either of these locations become compromised, these nerves can be pinched or otherwise damaged causing a sensation of pain and discomfort. [26].

\section{Mechanical Low Back Pain}

LBP is experienced by all age groups and is a problem growing alongside the world's population $[2,28]$. LBP is now the biggest factor in disability and is an everpresent challenge as it is difficult to understand the pathological cause in most cases [2, 28]. Fortunately for most, when they experience LBP it is acute and only lasts a few weeks. However, for chronic cases (those longer than three months), LBP is likely to worsen without some form of healthcare intervention. As LBP starts to cause serious problems, this ends up costing the patient not only in hospital and clinic visits, but in precious lost time due to disability.

\section{Common Diagnosis}

While the underlying cause of back pain is difficult to determine, many agree that most can be attributed to some form of mechanical complication [11]. These mechanical problems include strains and sprains of the muscles and tendons, disc degeneration, radiculopathy, spondylolisthesis, and stenosis among a handful of others. Focusing on the latter few, each of these complications tend to be from normal aging and repetitive misuse of the lower spine.

Degenerative disc disease (DDD) is largely seen as the most common cause of LBP and is an overarching factor in many other spinal complications. DDD is difficult to detect early on and is usually diagnosed after a patient has been seen for pain and imaging has been taken. Damage to discs can happen for any number of reasons, but is common among older patients, with some $85 \%$ of individuals over 50 showing evidence of DDD upon autopsy [29]. Typically, dehydration of the disc's NP is associated with DDD. This dehydration leads to more wear placed on the AF which then further deteriorates as loading is increased. At some point, the disc is no longer able to maintain sufficient height for nerve roots or motion to occur and the sensation of pain is radiated. The breakdown of the disc can also lead to disc herniation, bulging disc, and spinal stenosis.

Stenosis is a narrowing of the spinal column, foramen, or lateral recess. This condition can be caused by bone spurs, arthritis, disc degeneration, or trauma. As the openings for the nerve and spinal cord narrow, they are more likely to pinch down or irritate the nerve causing pain. 
Spondylolisthesis begins with a vertebra that slips forward, putting pressure on nerves as they leave the canal. This slippage usually happens at L5S1 as the high vertical and shear loads on the spine reach their peak in this area. Spondylolisthesis can come in many different forms, isthmic fracture, degenerative, and traumatic, but all result in pain stemming from the low back.

All of the previous conditions can be bundled under radiculopathy. Radiculopathy is a general term for conditions in which spinal nerve root is injured, inflamed, or compressed. Any form of this physical alteration of the nerve can lead to a pain or numbing sensation felt around the area of the body the impacted nerve serves [11].

\section{Current Conservative Treatment Options}

Conservative treatment for LBP usually begins with over-the-counter pain medication, activity limitation, and light stretching. As healthcare professionals become involved, stronger analgesics and physical therapy modalities are extensively used before resorting to spinal injections or surgery. Some of the basic physical therapy options are stretching and lumbar spine core strengthening (LSCS) exercises, water therapy, and light brace use.

Physical therapy includes a broad range of treatment options. Typical treatment options for LBP sufferers include yoga, stretching, manual therapy, or a McKenzie exercise approach all depending on the patient and clinician experience. No matter the physical therapy path, the goal is to reduce pain by alleviating pressure on the nerves and improve the patient's spine health through exercise [14].

Water therapy takes place in a pool of water where the buoyant force lessens the weight placed on the lumbar spine. This reduction in weight eases pressure off of the IVDs which helps to lower a patient's pain and aids in the diffusion of nutrients to the disc. Exercise in the pool is encouraged as controlled motion helps to increase core muscle strength. With a stronger core, pressure on the nerves within the lumbar back is alleviated and pain reduced.

Lumbar support braces, readily found over-the-counter, aim to provide relief by applying an intraabdominal force to offload some of the weight from the spine. These types of braces also typically constrain the user's lower back, keeping them in an upright position. Acting like a weightlifter's belt, lumbar bracing helps to prevent further damage from muscle strains and incorrect posture while the increased pressure aids in pain relief (Figure 2-2A).

Currently, there is only one external distraction brace designed for LBP on the market that competes with the DMO project. The VerteCore Lift sold online by VerteCore Technologies is shown in Figure 2-2B. This brace distracts the spine by utilizing two mechanical ratchets on the lateral sides of the body to jack apart two brace 


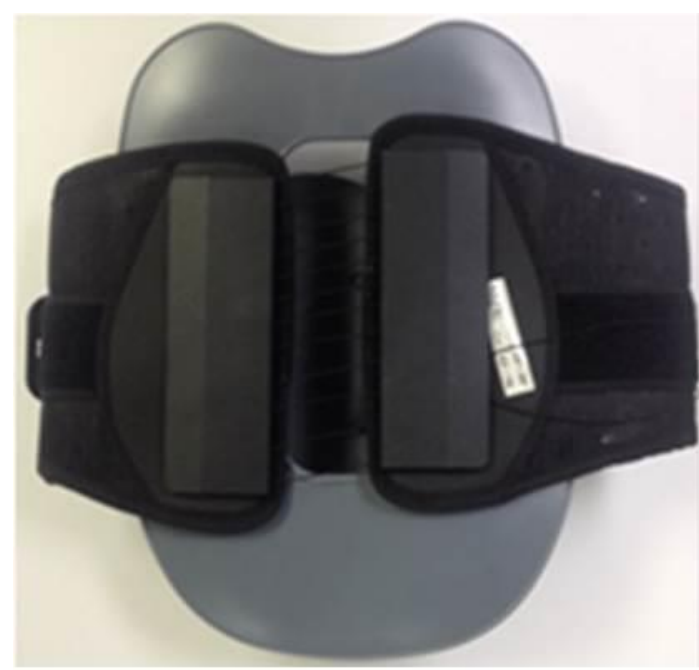

A)

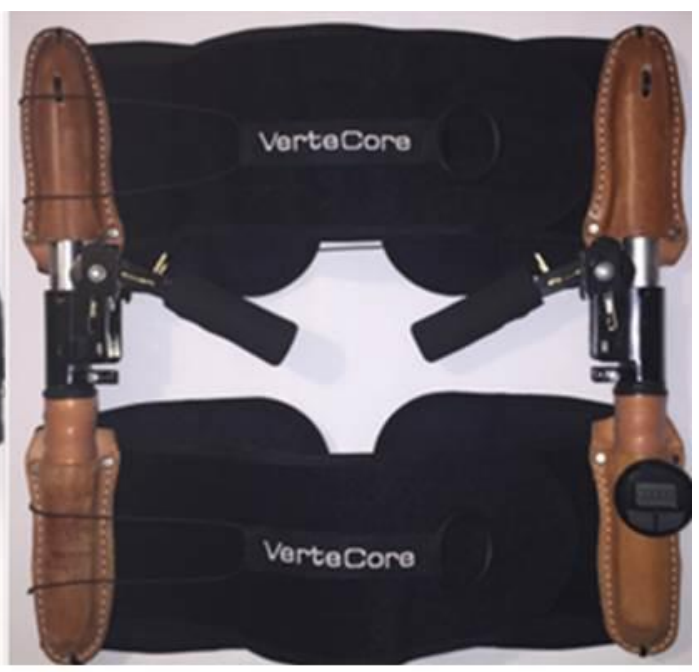

B)

Figure 2-2: Conservative LSO treatment options for LBP.

A) Conventional LSO (Cybertech 2000 -Biocybernetics International, CA, USA)

B) Mechanical LSO (VerteCore Lift - VerteCore Technologies, TX, USA) 
halves located on the hips and underneath the arms of the user. Like conventional LSOs, this brace limits lumbar motion, not allowing for rotation between the upper and lower halves of the brace components. A few other types of distraction braces have been developed by other groups, but they are unable to back up any scientific claims and have either been removed or did not make it to market.

\section{DMO Project}

The DMO project has been under development for a number of years with the main goal of offloading the lumbar spine while still allowing for lumbar spinal motion. This project is seen as supplemental to physical therapy- more accessible than water therapy and more effective than traditional braces currently on the market. For many individuals, pain is a limiting factor in successfully performing a complete round of physical therapy. In an attempt to relieve pain, the DMO offloads the spine, aiming to relieve any pinched nerves. With the added benefit of unconstrained motion, the patient may then complete their physical therapy pain free. Water therapy provides a similar function to the DMO due to the buoyant force on the body, but patients are required to attend sessions in a pool which may be an inconvenience not readily accessible to all individuals. Traction and inversion tables are another conservative option that offload the spine, but they restrain the user to the device and restrict motion while in use. Traditional LSOs do not allow for flexion, extension, rotation, or lateral bending of the lumbar spine attempting to protect the patient from instability. Unfortunately, reliance on this method of pain reduction may lead to muscle atrophy of the paraspinal and abdominals further deteriorating the condition. LSOs can also be described as uncomfortable and unnatural and only provide relief while being worn as they are unable to improve conditions within the back.

Wearing the DMO, however, patients can undergo motions necessary for daily living while offloading the forces placed on their vertebral bodies and compromised nerves. The distractive force applied by the DMO is postulated to relieve pressure within the IVDs, allowing nutrients to be diffused into the disc - restoring disc height and improving patient satisfaction even after the brace has been removed. The brace's low resistance to bending enables patients to utilize their own strength to perform activities, further improving their core muscles rather than deteriorating them.

\section{Design of the Previous DMOs}

The original concept design, DMO1, is shown in Figure 2-3A. This design was custom fitted to the designer and utilized a bent rod as the method for distraction. The rod created an inverted $U$ shape and was placed on the lateral sides of the brace. A caster placed on the upper portion of the DMO rode along the rod and provided distraction by cranking down and displacing the bent rod. DMO1 was limited to minimal sagittal plane motion as the bent rod would buckle, causing off axis forces and the caster to jam. 


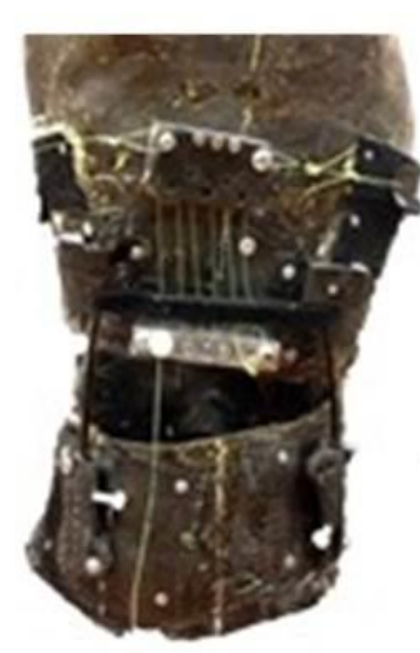

A)

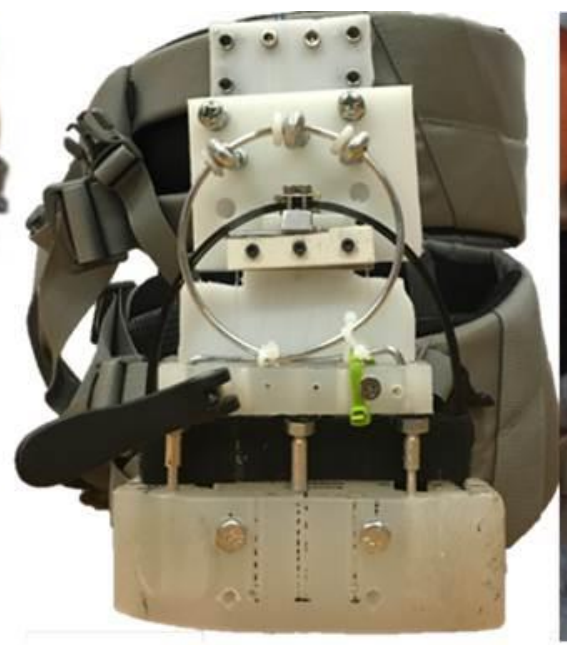

B)

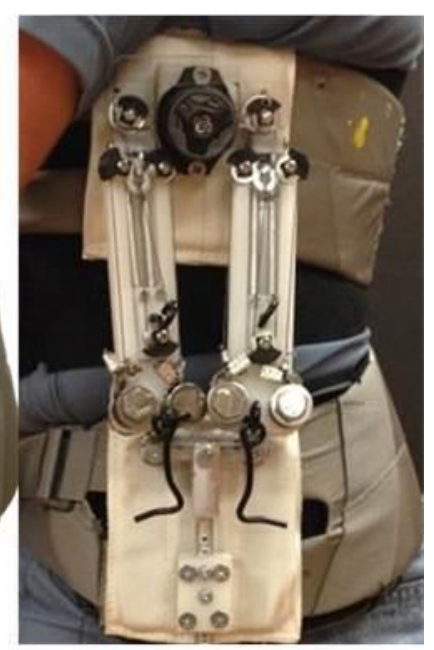

C)

Figure 2-3: Previous DMO prototypes.

A) DMO1 - Floating caster and bent rod with molded body engagements

B) DMO2 - Non-deformable ring and bent rod with belt style body engagements

C) DMO3 - Constant Force Springs with ball joints and belt style body engagements 
Nonetheless, DMO1 was successful in offloading the spine in upright stance and some motion in the sagittal plane [20].

DMO2 built upon the bent rod design (Figure 2-3B). While the bent rod remained the same, the caster was replaced with a guide ring that did not jam. While motion was still limited to the sagittal plane, DMO2 was fitted with customized hip belts and brought more versatility to the brace so that it could be tailored to multiple people [21].

DMO3 (Figure 2-3C) reworked the DFM. In place of a bent rod, DMO3 utilized four actuators powered by constant force springs. Two of the actuators were paired on either side of the brace and linked to the torso glove and pelvic belt by tie rod ends. Each pair pivoted on a rocker bar about a movable anchor point that would be situated about the rotational axis of the patient. By using tie rod ends and the rocker bar, more motion was accessible with the brace, enabling some axial rotation and a full $25^{\circ}$ in flexion. The constant force springs provided a balance between the paired actuators while the patient moved about [21]. This design was utilized in a clinical trial and the concepts were used as a starting point for DMO4.

\section{Design of the Testing Setup, Spine, and LARS}

In order to test the functionality of the DMO designs in a controlled setting, an RTP was utilized to capture and record forces and moments involved within the lumbar spine during DLAs with and without the DMO installed [20] (Figure 2-4). A human analog model was created to mimic the properties of an adult human torso and was attached to the RTP at either end by six-axis load cells. One of the load cells remained stationary on platform simulating loads through the lumbar spine and the other was programmed to move about, simulating motions necessary for DLAs.

The bulk of the model was made from a male mannequin and reinforced so that it would be able to withstand the forces applied through it. Attachments were made at the neck and just below the ribs for the load cell and biomimetic spine to be secured. The model was wrapped in textured material to mimic human skin tissue and provide engagement for the DMO. The biomimetic spine was created by molding the lumbar vertebrae of a human cadaver. IVDs were fabricated with urethane and tested to match the mechanical properties of human discs, about $0.66 \mathrm{Nm} /{ }^{\circ}$, per previously recorded cadaveric data $[19,20]$. The bottom of the biomimetic spine attached to the second load cell and was surrounded by the pelvic girdle portion of the mannequin. This portion of the mannequin allowed the DMO's hip belt to be secured without contacting the spine and lower load cell.

\section{DMO Testing Protocol}

To apply loading conditions to the spine and DMO, the RTP gimbal assembly was programmed to apply a vertical load to the spine and rotate about so that the torso moved 


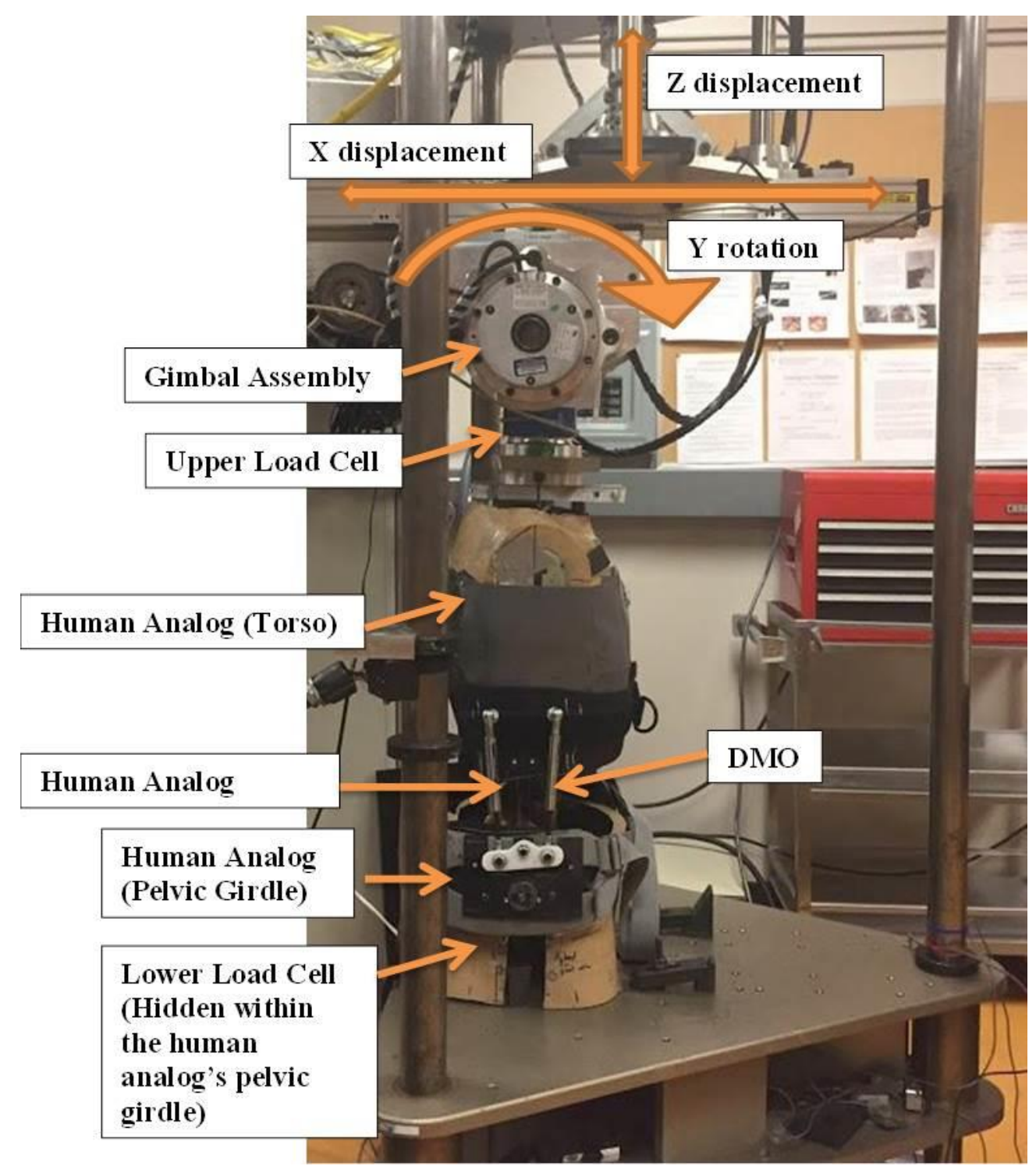

Figure 2-4: RTP with human analog model and DMO installed. 
in the sagittal plane. Beginning with a presumed Center of Rotation (CoR), the RTP balanced out any off-axis forces to the spine's true CoR by translating in the $\mathrm{x}$ and $\mathrm{z}$ directions. After the forces were within tolerance $( \pm 3 N)$, the gimbal rotated to the next half degree increment. This series of rotations and balancing was carried out until the limit for the test was reached $\left(25^{\circ}\right.$ in flexion and $10^{\circ}$ in extension) [19]. Each test was performed twice, once without the DMO, and once with the DMO installed. At each increment, the vertical and shear loads and bending moment applied to the spine and through the spine were recorded. For test runs including a DMO, the difference between these two values yielded the force and moment values for the DMO. 


\section{CHAPTER 3. BIOMECHANICAL EVALUATION OF A FULL-LENGTH (T12-S) SYNTHETIC LUMBAR SPINE MODEL ${ }^{1}$}

Over the years, the custom created biomimetic spine began to wear out and lose its mechanical stiffness properties. Before DMO4 could be tested on the RTP, a replacement model needed to be sourced and tested. This new model needed to fit within the constraints of the existing torso components, maintain its mechanical properties over many test conditions, and be within normal human range. Figure 3-1 shows the lab created spine and a commercially available synthetic T12S1 lumbar spine model from Sawbones Inc. (Product \#3430, Sawbones Inc., Pacific Research Laboratories, Vashon, WA). Unfortunately, the Sawbones biomechanical lumbar spine model had not been previously tested for its similarities to human cadaver spines, nor were the spines' local and global IAR locations known - necessary for prescribing an initial rotation point for the RTP during flexion and extension tests. With a similar sized model, L2L5, meeting human cadaver standards, the L1S1 biomechanical model was purchased and the requisite testing performed in house on the RTP. Utilizing infrared cameras and marker system along with a unique method for isolating segments for use in Crisco's IAR equation, each segment's path and IAR during flexion was recorded and compared to prior data.

\section{Introduction}

Cadaveric tissue-based testing is the standard method for biomechanically evaluating spinal instrumentation in the lumbar spine [30, 31]. In general, the motion response of each spinal segment is measured in response to a known loading condition. Application of pure moment loads is the most common [31,32] method and can also be modified with the addition of a compressive follower load [33,34] or in combination with compression and/or shear forces [35, 36]. More recently, multi-body synthetic lumbar spine models have been developed and are commercially available as a cost saving alternative to human cadaveric tissue (Sawbones Inc., Pacific Research Laboratories, Vashon, WA). The original validation studies of these synthetic models were done on either a single lumbar segment (L3-L4) model [37-40] or various multilevel lumbar models [41, 42]. Camisa et al. validated the flexion/extension, lateral bending, and axial rotation flexibility properties of a L1-L5 model against human cadaveric tissue tested under pure moment loading conditions [42]. Range of motion data were comparable to cadaveric specimens. Wang et al. studied the anatomical and biomechanical flexibility properties of a single L3-L4 synthetic lumbar model and also found no differences with human cadaveric data in the literature [40]. The model was further used by Wang et al. to compare different spinal fusion instrumentation under cyclic pure moment load endurance testing [43].

\footnotetext{
${ }^{1}$ Reprinted from final submission with open access permission. D. J. Diangelo, Hoyer, D., Chung, C., "Biomechanical evaluation of a full-length (T12-S) synthetic lumbar spine model," MOJ Applied Bionics and Biomechanics, vol. 3, 2019 [24].
} 


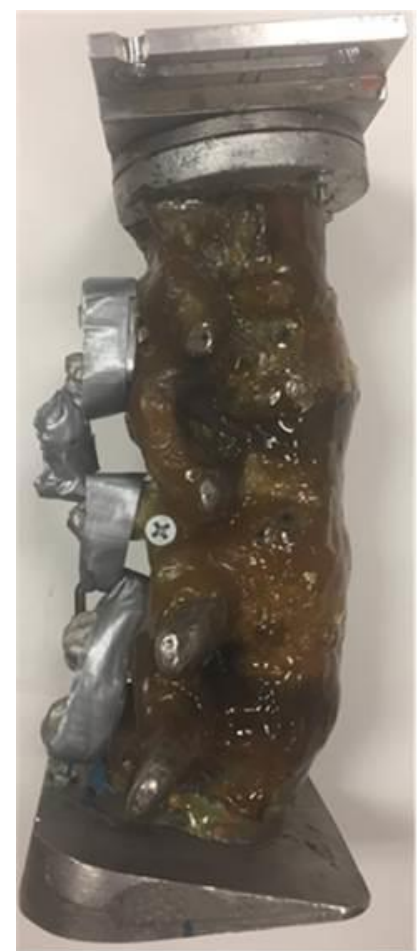

A)

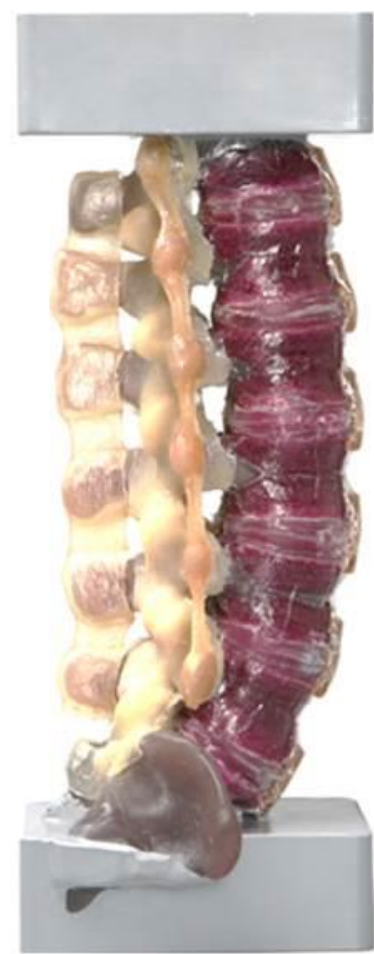

B)

Figure 3-1: Synthetic spines used in DMO testing.

A) Lab created biomimetic spine

B) Sawbones T12S1 synthetic biomechanical model (Sawbones \#3430, Pacific Research Laboratories, WA, USA) 
More recently, a full-length synthetic analog lumbar spine model (T12-sacrum) has become available for testing long multi-segment spinal constructs (Product \#3430, Sawbones Inc., Pacific Research Laboratories, Vashon, WA). Although these models can be used as an alternative method to tissue-based testing of spinal fusion instrumentation $[42,43]$, we have an interest to incorporate this full lumbar model into a testing protocol for biomechanically analyzing lower back support devices [44]. Regardless of the application, information of the rotational stiffness (angle versus moment) properties for this full lumbar synthetic model are not available. Additional kinematic parameters (like the center of rotation (CoR) and instantaneous axes of rotation (IAR)) will provide further biomechanical description of these devices. However, information of the IAR or CoR parameters for any of the synthetic lumbar spine models from Sawbones Inc. does not exist.

The CoR of lumbar spinal segments is typically calculated from the end range positions of the adjacent vertebrae while the IAR describes the moving path of the spinal segment using the positional changes of two fixed points on a rigid body [45-53]. The Reuleaux method is the most common technique used to calculate the IAR but is affected by the accuracy of the measurement system. To combat this, Crisco et al. [54] proposed a refinement to the Reuleaux method to reduce the error in the IAR calculation process, which was implemented in this study. The objectives of this study were to determine the flexion/extension flexibility properties of a full-length (T12-sacrum) synthetic lumbar spine model and to define the segmental and global kinematic parameters of each vertebral level of the model. The full-length synthetic lumbar spine model was tested under pure moment loading conditions and a modified version of Crisco's technique was developed to determine the global and local/segmental CoR and IAR kinematic parameters after the spinal segments were isolated into adjacent pairs. The long-term use for this synthetic spine model is to support the development of a standardized testing methodology for evaluating and designing lumbar spinal orthoses. In summary, this paper describes a new mathematical approach to determine the CoR and IAR parameters of a multi-level spine model using commonly measured spatial data of each vertebral segment. The technique was then used to describe these kinematic parameters for a new commercially available full length synthetic lumbar spine model. Additionally, standard pure moment biomechanical tests were carried out to quantify segmental motion and rotational stiffness properties of the synthetic model that were compared to in vivo and in vitro findings for validation purposes.

\section{Materials and Methods}

\section{Synthetic Lumbar Spine Model and Testing Platform}

A full-length (T12/S1) synthetic lumbar spine model (SKU\#3430, Sawbones Inc., Pacific Research Laboratories, Vashon, WA) was obtained with simulated intervertebral discs, vertebrae and ligaments. A photograph and schematic of the complete synthetic model are shown in Figure 3-2 and had potted blocks affixed to both ends. 


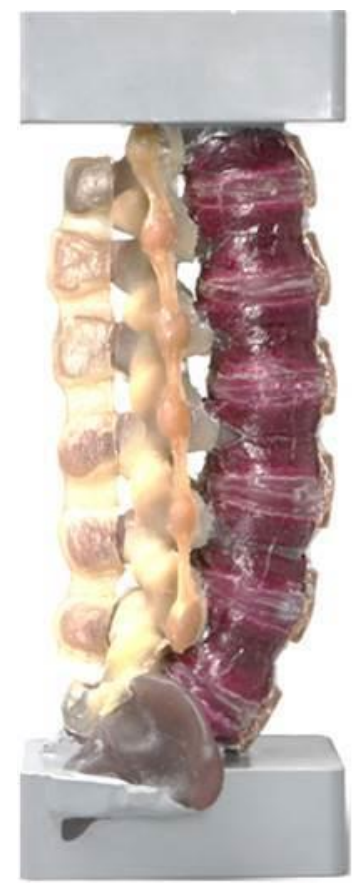

A)

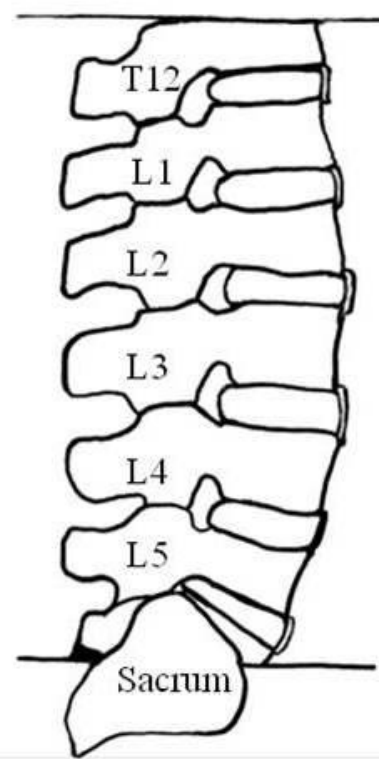

B)

Figure 3-2: Synthetic spine and schematic.

A) Synthetic lumbar spine model

B) Schematic representation of lumbar spine model

The sacrum served as the origin of global CoR and IAR parameters. 


\section{Kinematic Analysis}

The planar two-dimensional (2D) motion of each vertebral body of the model was measured using a camera-based system consisting of a receiver unit (two planar chargecoupled device (CCD) cameras) and active light-emitting diode (LED) targets (operating at near infrared light). An array of two LED targets, separated by 3 inches, was attached rigidly to each vertebral level (Figure 3-3). The location of the array in three-dimensional space uniquely determined the global position of each vertebra with respect to a fixed reference frame (i.e., the receiver unit) that was transferred to the sacrum using principles of rigid body mechanics. Local vertebral motion was expressed relative to the subjacent vertebral body using the equations and methods described below. A modified version of the Reuleaux method as proposed by Crisco et al. [54] was used to calculate the CoR and IAR parameters.

\section{Method for Analyzing Multi-Level Spine Model Segmentally}

In order to determine the local IAR of each spinal level, two adjacent spinal bodies were isolated from the full spine model. The motion of the spinal segment's upper body (UB) had to be expressed relative to the stationary lower body (LB) by negating all translational and rotational changes due to the motion of the lower body from both the UB and LB. Figure 3-4 and the following equations define the method. The known (global) locations of each body are relative to the sacrum target array after being transferred from the reference frame of the camera. First, a vector UB2' was defined as the spatial position of the UB at time point 2 due to the motion of the lower body alone (i.e., as if the UB was rigidly attached to the LB). The spatial position (Cartesian coordinates of $x$ and y) of UB2' was calculated using Equation 3-1A-E. This location was expressed as the sum of the starting linear position of the UB at time 1 and the linear displacement of the LB between time points 1 and 2, minus the linear displacement of the UB due to the rotation of the LB between time points 1 and 2 .

$$
\begin{gathered}
\overrightarrow{\Delta_{12} L B}=\overrightarrow{L B_{2}}-\overrightarrow{L B_{1}} \\
\overrightarrow{U B_{2^{\prime}}}(x)=\overrightarrow{U B_{1}}(x)+\overrightarrow{\Delta_{12} L B}(x)-L *\left[\cos \left(\beta_{1}\right)-\cos \left(\beta_{2}\right)\right] \\
\overrightarrow{U B_{2^{\prime}}}(y)=\overrightarrow{U B_{1}}(y)+\overrightarrow{\Delta_{12} L B}(y)-L *\left[\sin \left(\beta_{1}\right)-\sin \left(\beta_{2}\right)\right] \\
\beta_{1}=\tan ^{-1}\left(\frac{U B_{1}(y)-L B_{1}(y)}{U B_{1}(x)-L B_{1}(x)}\right) \\
\beta_{2}=\beta_{1}+\Delta_{12} L B(\theta)
\end{gathered}
$$

The angular $(\Theta)$ or rotational position UB2' was calculated using Equation 3-2. The angle of UB2' was expressed as the sum of the angular position of the UB at time point 1 and the angular displacement of the LB between time points 1 and 2 . 


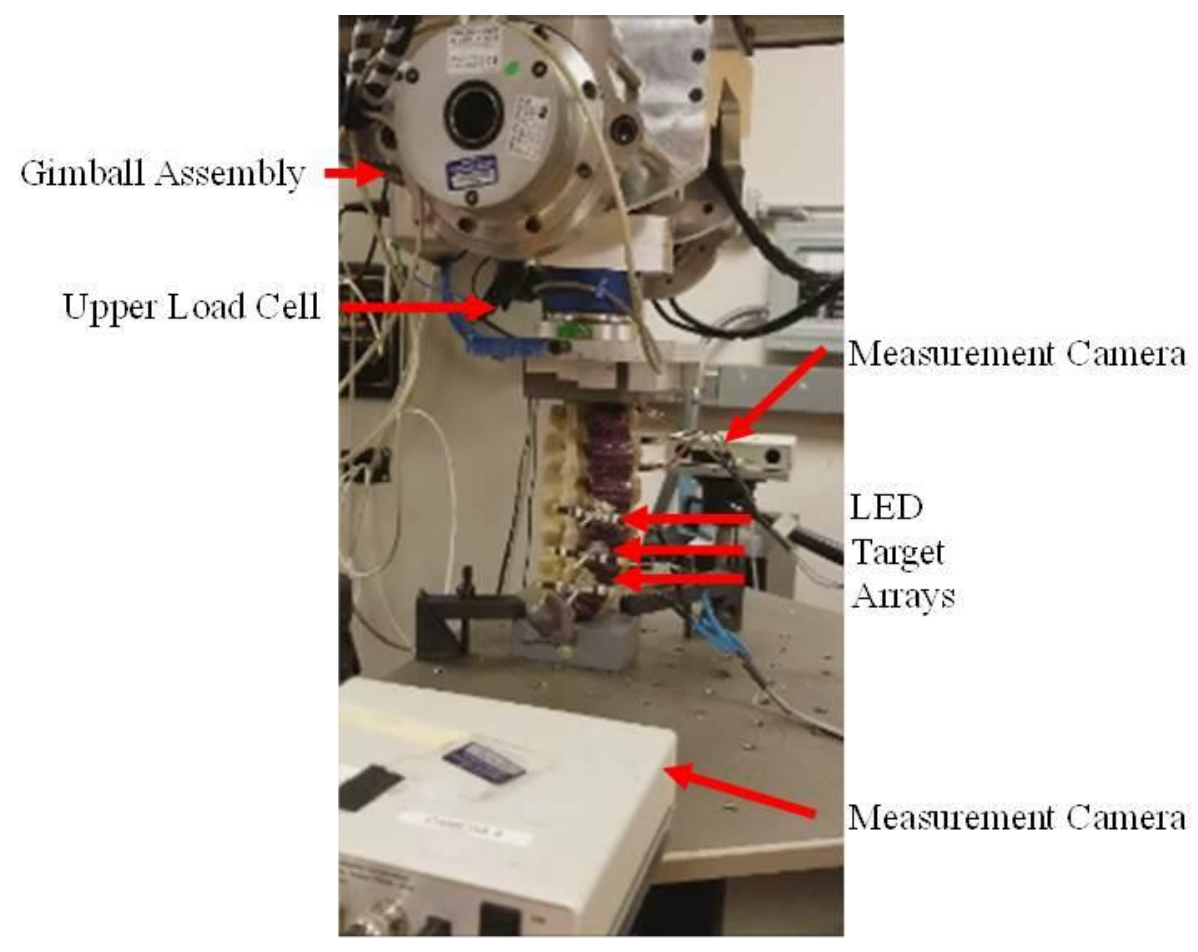

Figure 3-3: Experimental set-up of a synthetic lumbar spine mounted in the robotic testing platform. 


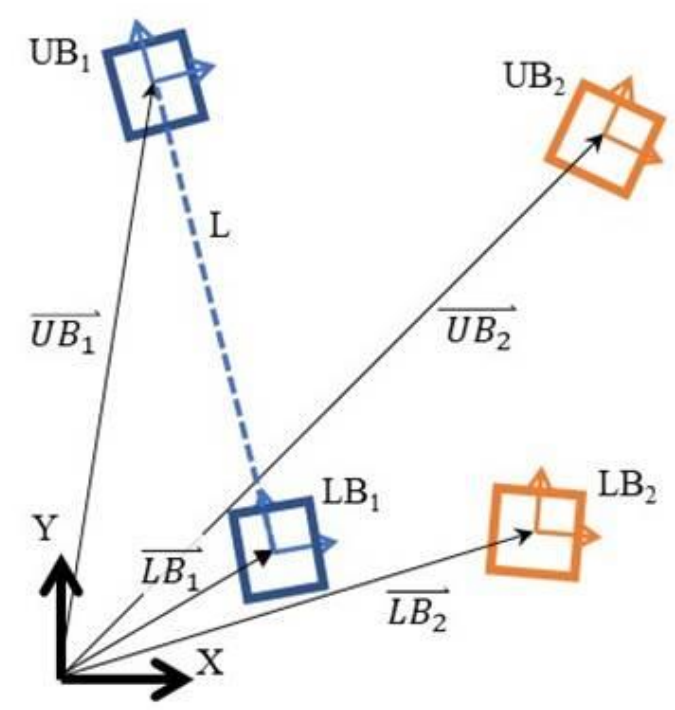

A)

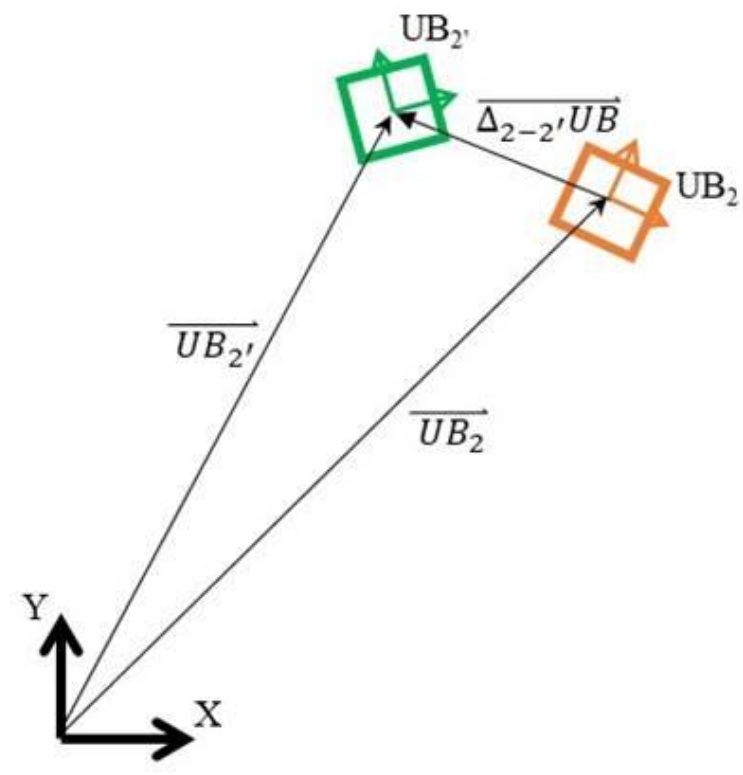

C)

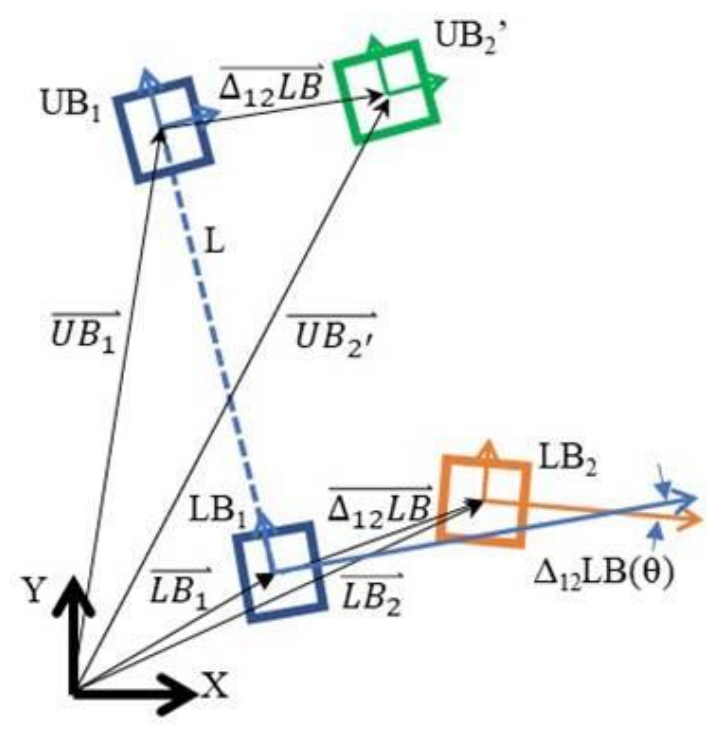

B)

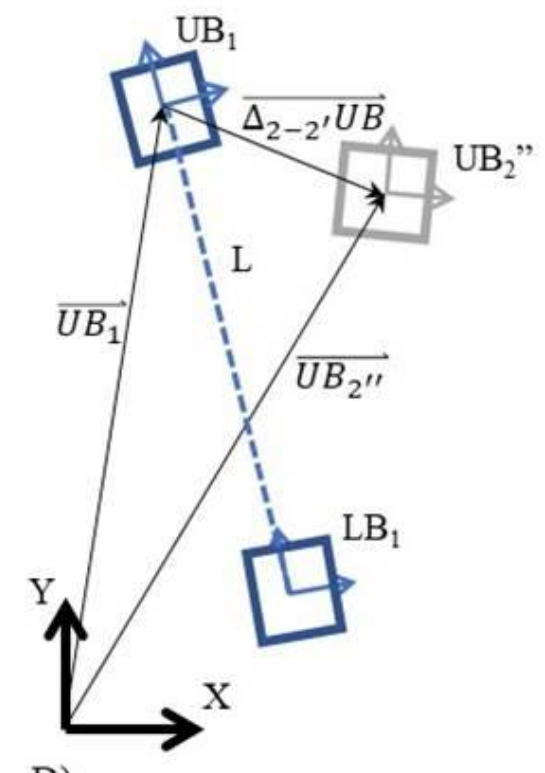

D)

Figure 3-4: Method for determining the local IAR of upper body (UB) relative to the lower body (LB).

A) UB1 is a known location of Upper Body at time 1, UB2 is a known location of Upper Body at time 2, LB1 is a known location of Lower Body at time 1, LB2 is a known location of Lower Body at time 2.

B) UB2' is a calculated location of Upper Body at time 2 due to the motion of Lower Body alone.

C) The relative change of the UB between UB2' and the final position UB2 is given by $\triangle 2-2$ 'UB.

D) UB2"' is a calculated location of Upper Body at time 2 due the motion of Upper Body alone relative to a stationary LB. 


$$
U B_{2^{\prime}}(\theta)=U B_{1}(\theta)+\Delta_{12} L B(\theta)
$$

Finally, a second vector UB2" was defined as the spatial position of UB at time point 2 due to the motion of the UB alone (i.e., the effects of the motion of the LB are negated). UB2" was calculated using Equation 3-3A-B. The position of UB2"' is calculated using the known position of UB2 minus the computed position of UB2', plus the known starting position of UB1,

$$
\begin{aligned}
& \overrightarrow{\Delta_{2-2}, U B}(x, y, \theta)=\overrightarrow{U B_{2}}(x, y, \theta)-\overrightarrow{U B_{2 \prime}}(x, y, \theta) \\
& \overrightarrow{U B_{2^{\prime \prime}}}(x, y, \theta)=\overrightarrow{U B_{1}}(x, y, \theta)+\overrightarrow{\Delta_{2-2^{\prime}} U B}(x, y, \theta)
\end{aligned}
$$

These calculations were repeated incrementally every 0.5 degree to determine a new path of motion for UB relative to a fixed LB reference frame so that Crisco's equations [54] could be used to calculate the local CoR and IAR parameters segmentally across the different levels of the synthetic spine. The global IAR values of each spinal segment was calculated every 2 degrees change in 0.5 degree increments (i.e., 0-2, 0.5$2.5,1.0-3.0$, etc.)

\section{Testing Protocol}

The synthetic spine model was mounted in a robotic testing platform [19] that had four programmable degrees of freedom with a positional resolution of $2 \mu \mathrm{m}$ in $\mathrm{x}, 0.31 \mu \mathrm{m}$ in $\mathrm{z}$, and $0.0002^{\circ}$ about y (Figure 3-3). An upper load cell (ULC) (JR3 Inc., Woodland, CA) with $400 \mathrm{~N}$ load and $40 \mathrm{Nm}$ moment capacities was attached to the gimbal assembly and measured the forces and moment applied to the spine model at a rate of $15 \mathrm{~Hz}$. The spine model was tested under a pure moment and an axial load of $20 \mathrm{~N}$ was applied to the spine [55] and rotated between $25^{\circ}$ flexion and $10^{\circ}$ extension at $1.6^{\circ} / \mathrm{s}$. Motion in either direction was stopped if the moment limit exceeded $10 \mathrm{Nm}$. The axial load was applied through the global point of rotation of the entire spine model (i.e., point of rotation of T12 relative to the sacrum). This step minimized any off-axis bending moment from being created by the axial load. The control strategy has been validated and used in other biomechanical studies [44, 55-57].

\section{Data Management}

The global stiffness of the synthetic spine model was expressed as a function of the applied moment relative to the overall global rotation. The position data of each vertebral body were measured at $10 \mathrm{~Hz}$ and used to determine the local and global IAR and $\mathrm{CoR}$ parameters. Noise in the camera data was filtered out using a curve fitting approach. The rotational response of each Motion Segment Unit (MSU) level was normalized to the total rotation of the synthetic spine model and expressed as a percent contribution (\%Rot). Similar rotational data sets from both in vivo studies [58-63] and in 
vitro pure moment tests $[32,36,64]$ on cadaveric (L1-S1) lumbar spines were compiled for comparison purposes.

\section{Results}

\section{Global Stiffness and MSU Rotational Contribution}

The flexion and extension moment - rotation data of each vertebral level of the synthetic model is shown in Table 3-1. The spine model reached $25^{\circ}$ flexion at a moment of $7.4 \mathrm{Nm}$ and $10^{\circ}$ extension at a moment of $6 \mathrm{Nm}$. However, since only validation data for the L2-L5 synthetic models exists in the Sawbones literature [41], the equivalent segment data from the full-length synthetic spine model was extracted and compared. The corresponding stiffness curve in Figure 3-5 shows the similarity between the two data sets. To further validate the structural properties of the full-length synthetic model, the rotational response of each vertebral level of the full-length (T12-S1) model was normalized to the total lumbar spine rotation and compared to similar in vivo and in vitro data sets found in the literature. Figure 3-6 compares the percent motion contribution of the synthetic model to three human cadaveric data sets $[32,36,64]$ tested under similar pure moment loading conditions, while Figure 3-7 compares the full-length data to five in vivo studies [58-63]. No statistical analysis was performed, as data from only one fulllength analog spine was available for comparison. However, as illustrated in

Figures 3-6, 7, all spinal levels were involved in contributing to the overall motion response regardless of the testing method (i.e., in vitro, in vivo, or synthetic model), and a close match was found at the three upper motion segment units. The mean and standard deviation of the three pure moment in vitro studies was added to Figure 3-6 and the mean and standard deviation of the five in vivo studies was added to Figure 3-7. The \% Rot of all MSU levels was within 2 standard deviations for either test comparison.

\section{Global CoR and IAR Patterns}

The global path of the instant axis of rotation of each vertebral body relative to a fixed sacrum reference frame is shown in Figure 3-8 for the full sweep of motion from $10^{\circ}$ extension to $25^{\circ}$ flexion. The transition point defining neutral orientation $\left(0^{\circ}\right)$ is also included. The IAR path of the upper four vertebral bodies was located in the mid-region of the bodies and transition downward during flexion, while the IAR of the lower two (L4 and L5) vertebral bodies transitioned to a horizontal path that moved posteriorly during flexion. Minimal translation of the IAR path occurred during extension. When looking at the relative location of the IAR path for each body relative to the fixed reference frame of the sacrum, the path was located close to the mid-distance of each body relative to the sacral body. That is, the IAR path of T12 body was located within the L3 body, starting at the top surface and moved downward, which is about half the distance between the T12 and $\mathrm{S} 1$ bodies. The location of the global IAR paths of the other vertebral bodies was 
Table 3-1: Flexion and extension response per motion segment unit.

\begin{tabular}{lcc}
\hline Motion Segment Unit & Flexion (Deg) at 7.4Nm & Extension (Deg) at 6Nm \\
\hline T12-L1 & 5.4 & 1.5 \\
L1-L2 & 2.3 & 1.5 \\
L2-L3 & 4.5 & 1.5 \\
L3-L4 & 3.3 & 1.5 \\
L4-L5 & 6.3 & 1.5 \\
L5-S1 & 3.3 & 1.6 \\
T12-S1 & 25.1 & 9.1 \\
\hline
\end{tabular}

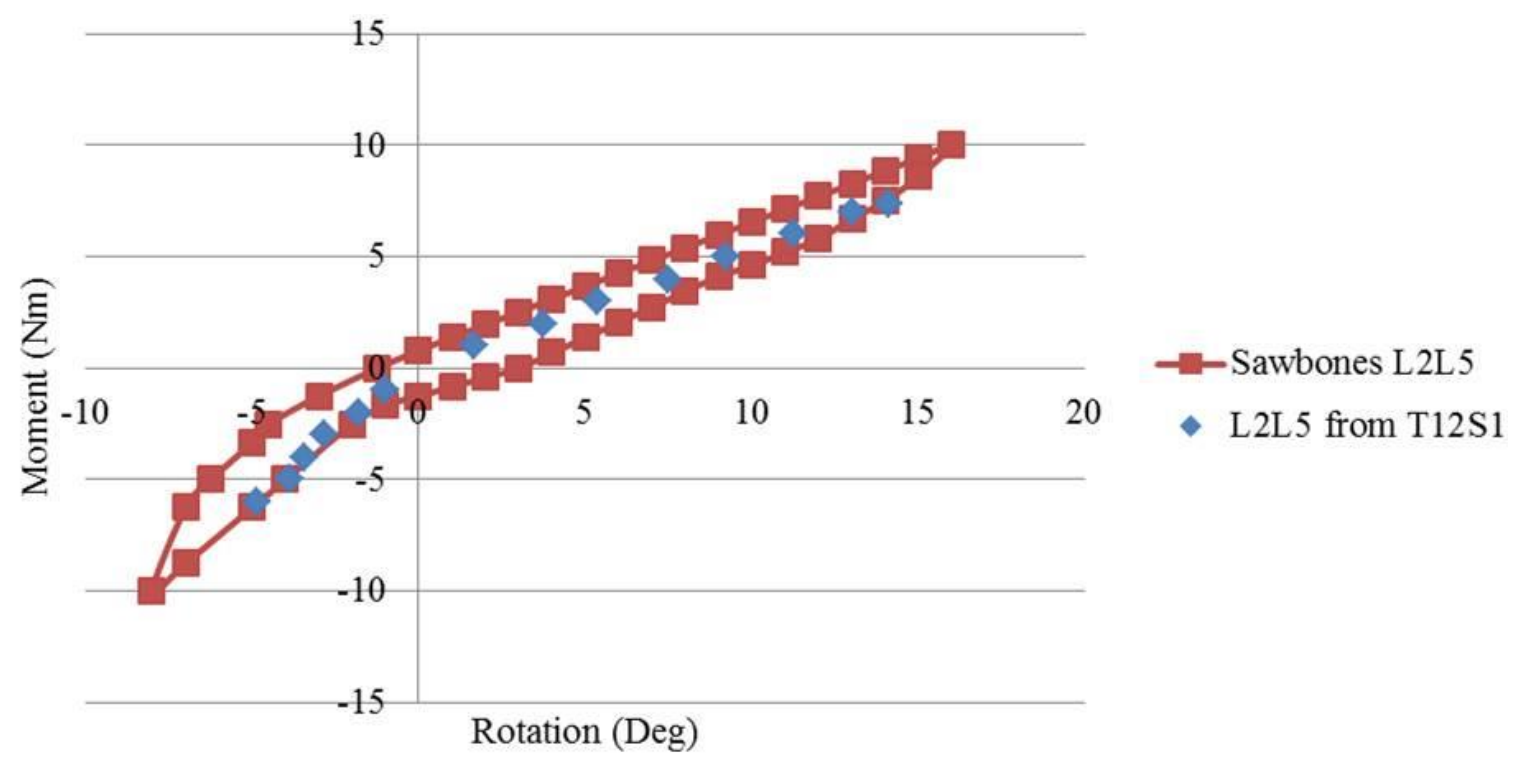

Figure 3-5: Comparison of moment-rotation stiffness properties.

The rotational stiffness of the L2-L5 Sawbones' Inc. model in red provided a comparison for the same segments of the previously untested full-length (T12-sacrum) model in blue. Data Source: J. Campbell, Imsdahl, S, Ching, RP, "Evaluation of a Synthetic L2-L5 Spine model for biomechanical testing," ed. Poster Presentation: Canadian Biomechanics Society, 2012 [41]. 


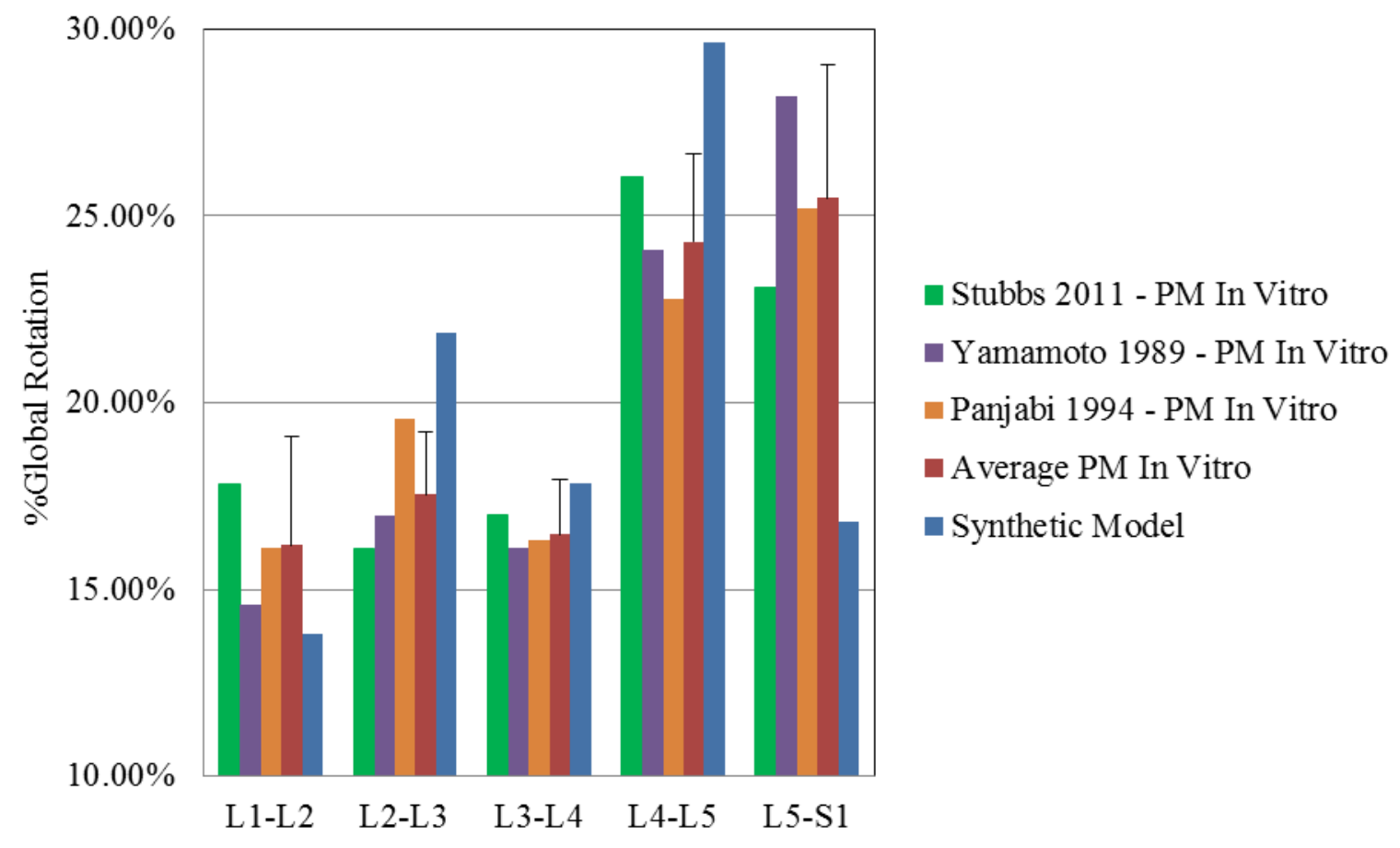

Figure 3-6: Comparison of percent global segmental motion response between synthetic model and in vitro pure moment test results.

Data Sources: M. M. Panjabi, et al., "Mechanical behavior of the human lumbar and lumbosacral spine as shown by three-dimensional load-displacement curves," The Journal of Bone and Joint Surgery, vol. 76(3), pp. 413-24, 1994 [32].

J. R. Stubbs, "Use of a multi-axis robotic testing platform to investigate the sagittal mechanics of the multi-body lumbar spine," Thesis and Dissertations (ETD), The University of Tennessee Health Science Center, 2011 [36].

I. Yamamoto, et al., "Three-dimensional movements of the whole lumbar spine and lumbosacral joint," Spine, vol. 14(11), 1989 [64]. 


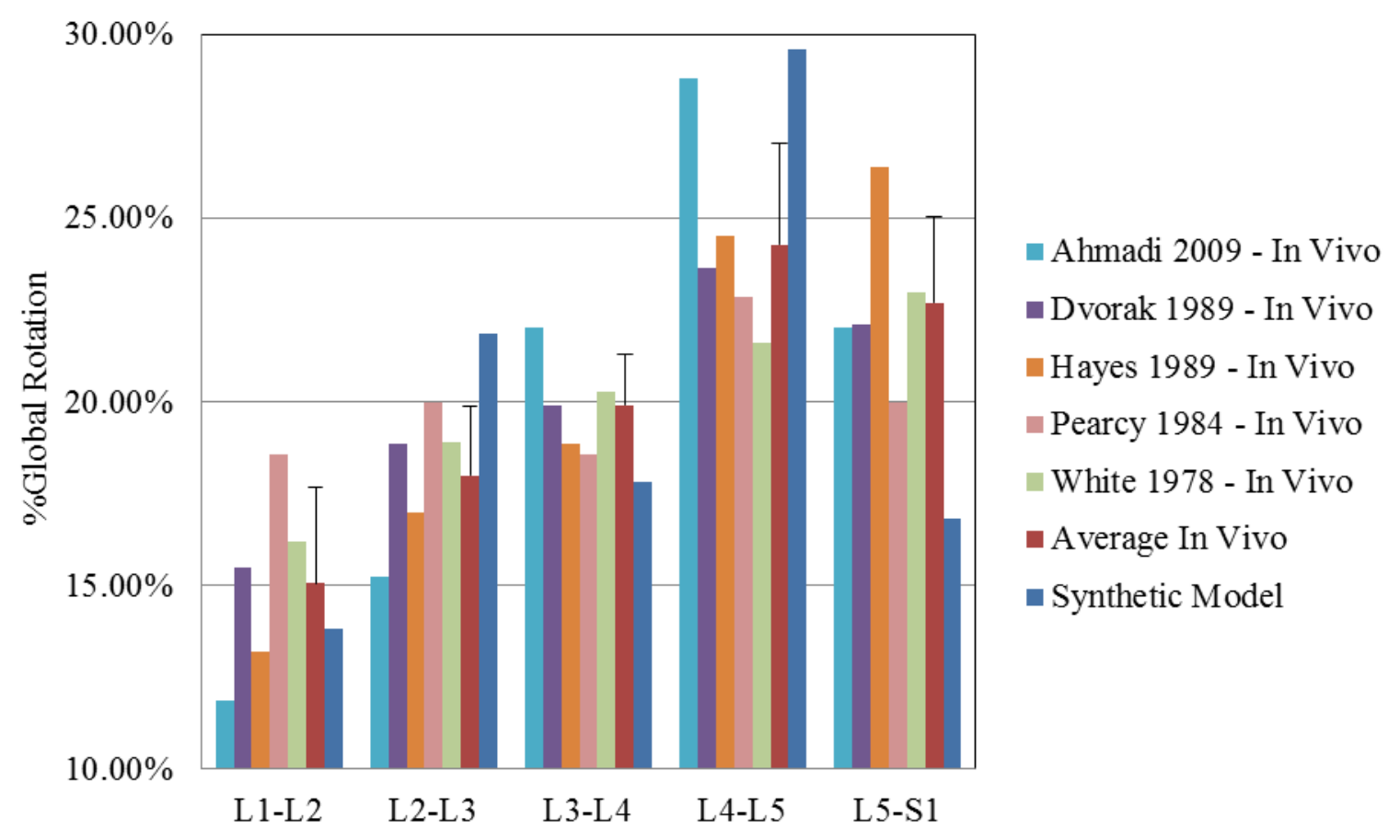

Figure 3-7: Comparison of percent global segmental motion response between synthetic model and in vivo motion patterns.

Data Sources: A. Ahmadi, Maroufi, N, Behtash, H, Zekavat, H, Parnianpour, M, "Kinematic analysis of dynamic lumbar motion in patients with lumbar segmental instability using digital videofluoroscopy," Eur Spine J., pp. 1677-85, 2009 [58].

J. Dvorak, Panjabi, MM, Chang, DG, Theiler, R, Grob, D., "Functional radiographic diagnosis of the lumbar spine. Flexion-extension and lateral bending," Spine, pp. 562-71, 1991 [59].

M. Hayes, Howard, TC, Gruel, CR, Kopta, JA, "Roentgenographic evaluation of lumbar spine flexion-extension in asymptomatic individuals," Spine, vol. 14(3), pp. 327-31, 1989 [60].

S. Lee, Wong, KW, Chan, MK, Yeung, HM, Chiu, JL, Leong, JC, "Development and validation of a new technique for assessing lumbar spine motion," Spine, vol. 27(8), pp. 215-20, 2002 [61].

M. Pearcy, Portek, I, Shepherd, J, "Three-dimensional x-ray analysis of normal movement in the lumbar spine. ," Spine, vol. 9(3), pp. 294-7, 1984 [62].

A. White, Panjabi, MM, Clinical biomechanics of the spine, 1990 [63]. 


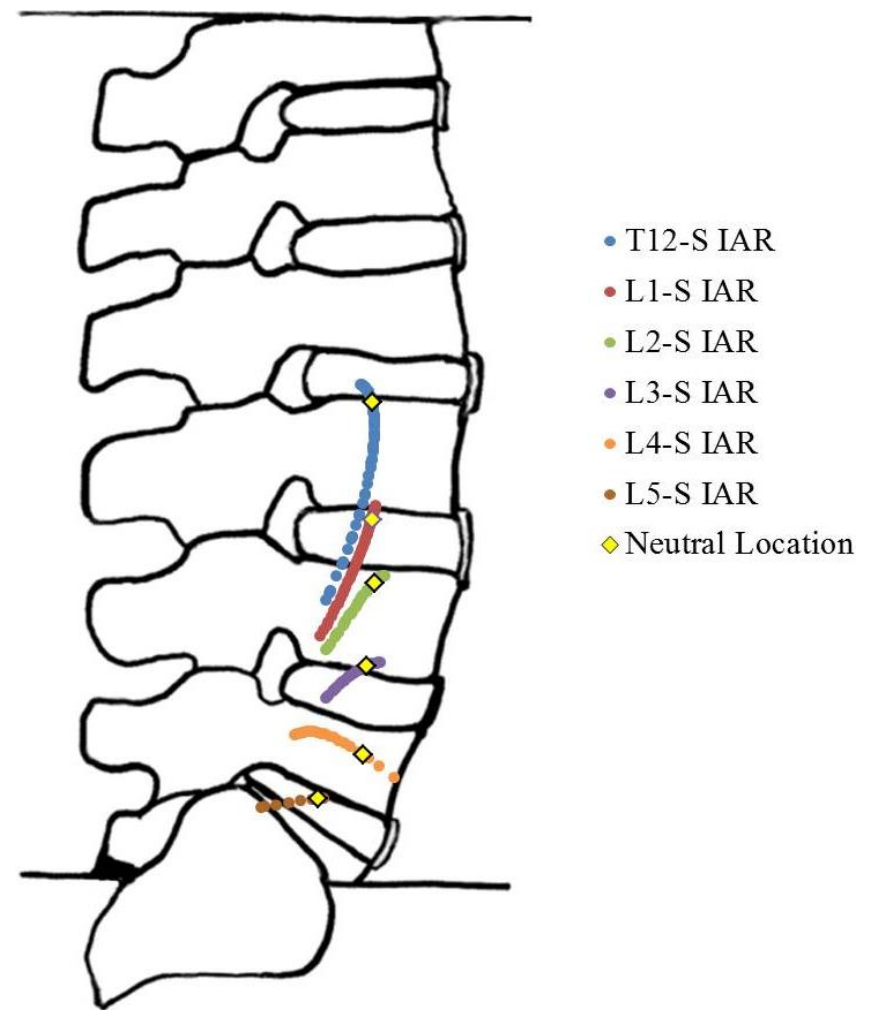

Figure 3-8: Global IAR patterns of each spinal level. 
similarly located close to the mid-distance between the respective vertebral body and the fixed sacral body.

\section{Local CoR and IAR Patterns}

The local IAR path of each vertebral body relative to its subjacent body is shown in Figure 3-9. For all MSUs, the local IAR paths were generally located along the superior endplate surface of the lower body. The local IAR of the upper two MSU (T12L1 and L1-L2) was near the posterior wall of the endplate surface while the local IAR locations of the remaining vertebrae were closer the midline of the end plate surface of the lower vertebral body. The profile of each MSU path is largely dictated by the mechanical properties of the interconnecting vertebral disc material and pseudo-ligament structures. The CoR values of each MSU are also shown on Figure 3-9 and were located near the mid-point of their respective IAR path. For those MSU with their CoR located near the midline of the vertebral disc, involvement of the facet joints was likely minimal.

\section{Discussion}

A unique set of biomechanical data describing the performance of a new commercially available full-length synthetic model of the lumbar spine is provided. However, before such models can be considered as an alternative material to human cadaveric tissue, validation of their mechanical properties is required. The global stiffness property was comparable to published in vitro findings found in the literature $[32,36,64]$ when tested under similar loading conditions. Similarly, the involvement of each motion segment was comparable to rotational patterns observed during in vivo flexion and extension activities. Since the published segmental rotations of L2-L5 model [41] were within one standard deviation of human cadaveric tissue, and the response of the full-length synthetic model was comparable to them, the full-length synthetic model can also be considered an adequate representation of human tissue. Further, in addition to establishing the biomechanical properties of the synthetic lumbar spine, a method was established to determine the local paths of the CoR of each MSU. This method can easily be implemented by other researchers capturing global positional data of each vertebral body.

For the synthetic lumbar spine model, the locations of the local CoR were close to the midline of the superior end plate of the lower subjacent body. Other researchers have also analyzed the IAR and CoR of the lumbar spine. Gertzbein et al. [46] biomechanically tested five human cadaveric L4-L5 segments and reported a locus of IAR points in the posterior half of the intervertebral disc region, while Haher et al. [47] found the IAR of a single L4-L5 segment to be in the vicinity of the facet joints. However, many of the earlier studies, similar to Gertzbein et al. [46] and Haher et al. [47], had methodological differences that limited direct comparison or validation to them. 


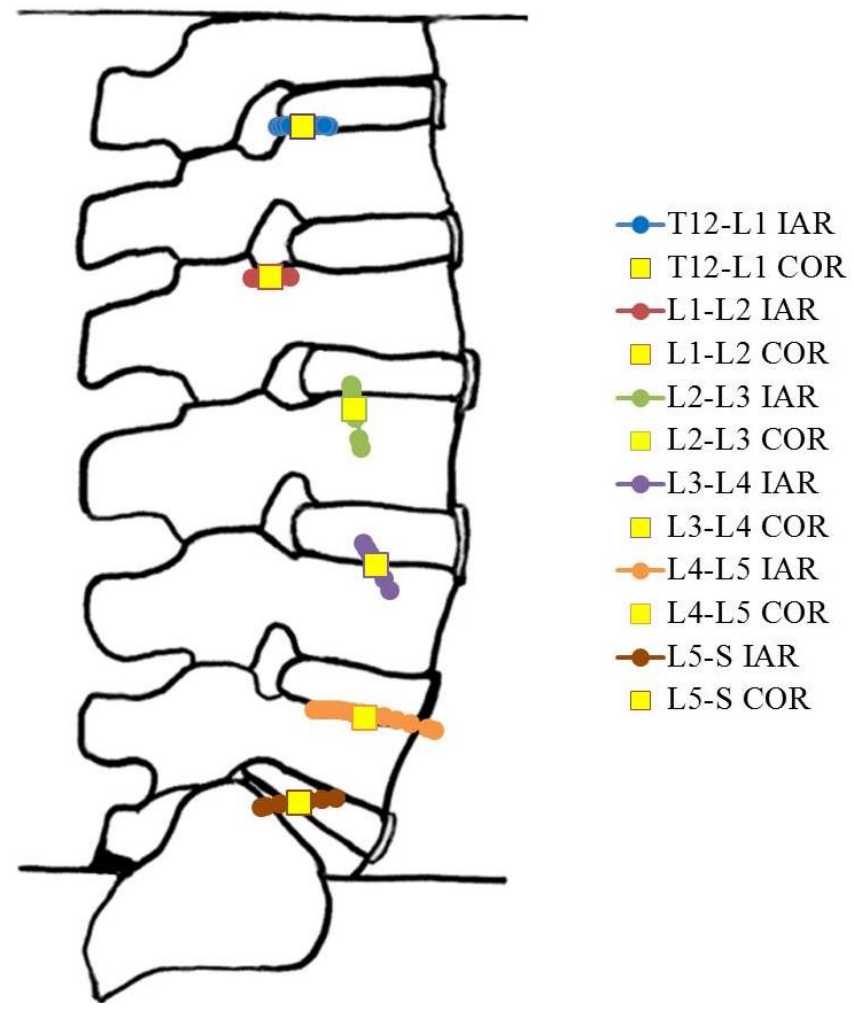

Figure 3-9: Local CoR and IAR patterns of each motion segment unit. 
Pearcy et al. [50] carried out one of the first in vivo studies measuring the IAR of the lumbar spine that addressed many of the potential error problems inherent with the measurement process itself. The mean location of the IAR positions for 10 subjects was close to the midline of the lumbar spine near the superior endplate of the lower vertebral body very similar to the IAR locations of the synthetic lumbar spine. Inoue et al. [49] carried out a biomechanical study on five deer L5-L6 segments to determine the effects of spinal instrumentation on the location of the IAR for different planes of motion, including the sagittal plane. Although the deer specimen differs from the human cadaver spine, the location of the IAR was in the region of the spinal cord, similar to L1-L2 segment of the synthetic spine. Of interest with their study was the method used to determine the IAR, which was similar to the approach used here.

More recent work by Bifulco et al. [65] used advanced video fluoroscopy methods to sequentially measure the IAR of a L2-L3 segment on the human subjects. The mean location of the IAR path was in the lower L3 vertebral body just posterior to the midline and near the upper end plate, very similar to the location of (L2-L3) of the synthetic spine model. Similarly, Liu et al. [66] used combined dual fluoroscopic imaging system with MRI imaging techniques to investigate the dynamic flexionextension motion of the lumbar spine in seven healthy human subjects doing a weightlifting activity. The average CoR location for L4-L5 was near the back one-quarter of the disc and close to the posterior edge of the disc for L5-S1, which is comparable to what was found for the COR locations of the synthetic spine model at those spinal levels.

This research is not without limitations. The pure moment protocol used here was unable to match the $2000 \mathrm{~N}$ axial load employed by Campbell et al. [41] to analyze single motion segment units. A $20 \mathrm{~N}$ axial load was applied to simply maintain compression on the spine for the duration of the pure moment testing method and only comparisons were made for flexion and extension. A sample size of one synthetic lumbar model was used for this testing, and no statistical analysis was performed on the outcomes. The likelihood of the mechanical properties of other models being similar is a dependent on the manufacturer. Never-the-less, each model can serve as its control group if used to test multiple spinal instrumentation systems. Further, the model analyzed in this study will be used to develop a biomechanical testing protocol for assessing spinal orthoses, where a repeatable material with a physiological response is desired. Knowledge of the location of the global IAR is required as input information for the control strategy of the robotic testing platform $[19,44]$.

\section{Conclusion}

Tissue-based testing methods are recognized as the standard of practice for analyzing and comparing spinal instrumentation devices. However, there are many limitations with using human tissue that can impact the feasibility and study outcome that include, but are not limited to, cost, tissue quality, age, gender, and surgical or disease condition. Synthetic biomimetic models of the human body provide a cost effective alternative to cadaveric tissue that should overcome many of these limitations. This study 
determined the rotational and biomechanical stiffness properties of a full length synthetic lumbar spine model and found them to be comparable to in vivo and in vitro findings supporting its use as a viable alternative to cadaveric tissue. Additionally, a new mathematical approach was developed to calculate the CoR and IAR parameters using commonly measured spatial data of individual bodies moving in space. The technique was used to determine these kinematic parameters of CoR and IAR for the full length synthetic lumbar spine model and were also found to be comparable to in vitro and in vivo published findings further support its use other biomechanical studies of the lumbar spine. Future plans are to use the synthetic lumbar spine model as the foundation of a testing standard for biomechanically assessing, classifying, and ranking low back support devices, as the use of human cadaveric tissue is not feasible. 


\section{CHAPTER 4. DESIGN OF DMO4}

\section{Design Goals of DMO4}

The overall design goal of DMO4 (Figure 4-1) was to improve the wearability found in previous generations while maintaining or improving spinal offloading capacity and ROM. DMO4 also needed to better conform to patient's anatomy and be easy to fabricate. The individual design goals for $\mathrm{DMO} 4$ were the following:

A. Effective Body Engagement. The DMO4 prototype was designed so that the pelvic belt remains secured to the hips and the torso belt snuggly wrapped around the torso while exercise and DLAs are performed. DMO3's pelvic belt was successful, but the torso belt lacked the ability to fully conform to the upper body.

B. Distractive Force Mechanism. The distractive force mechanism (DFM) was designed to provide a distractive force between the two body engagement belts. DMO4's force limit was based on previous clinical observations and a minimum force was set to $150 \mathrm{~N}$, about half of the upper torso weight of an average male. This distractive force was to be active throughout all ROMs.

C. Mobility-enabling component. DMO4 should be designed such that the allowed ROM matches the minimum required for typical DLAs and exercises. The target $\mathrm{ROM}$ of DMO4 was set to $25^{\circ}$ in flexion, $10^{\circ}$ in extension, and $10^{\circ}$ of axial rotation.

\section{DMO4 Body Engagement}

Figure 4-2 shows the body engagement features of DMO4. The hip belt component of DMO4 was largely unchanged from DMO3 as the Osprey Isoform Men's Hipbelt (Osprey Packs, Inc., CO, USA) provided solid engagement to the body. These hip belts are made of moldable foam (1) and can be anatomically fit to most patients. Nylon web strapping and a buckle (2) secures the hip belt to the body. Modifications were made to the DFM attachment while the body interface remained untouched. This modification added a pocket (3) to the lateral sides of the belt which allowed the DFM attachment to be integrated into the belt as opposed to being strapped together like in previous models.

The torso belt of DMO3, a second Osprey hipbelt, was exchanged for a belt based on a design from a conventional LSO (Figure 2-2A) with a back cinching pulley mechanism (4). This pulley mechanism allows the belt to be snuggly attached to the body via a pull tab (5) that wraps around the belt. Utilizing a combination of UBL and PowerMesh material alongside the cinching mechanism, the torso belt better conformed to patient anatomy. Similarly to the hip belt, the DFM attachment was integrated into the torso belt by adding components into a pocket (6) within the lateral sides. 


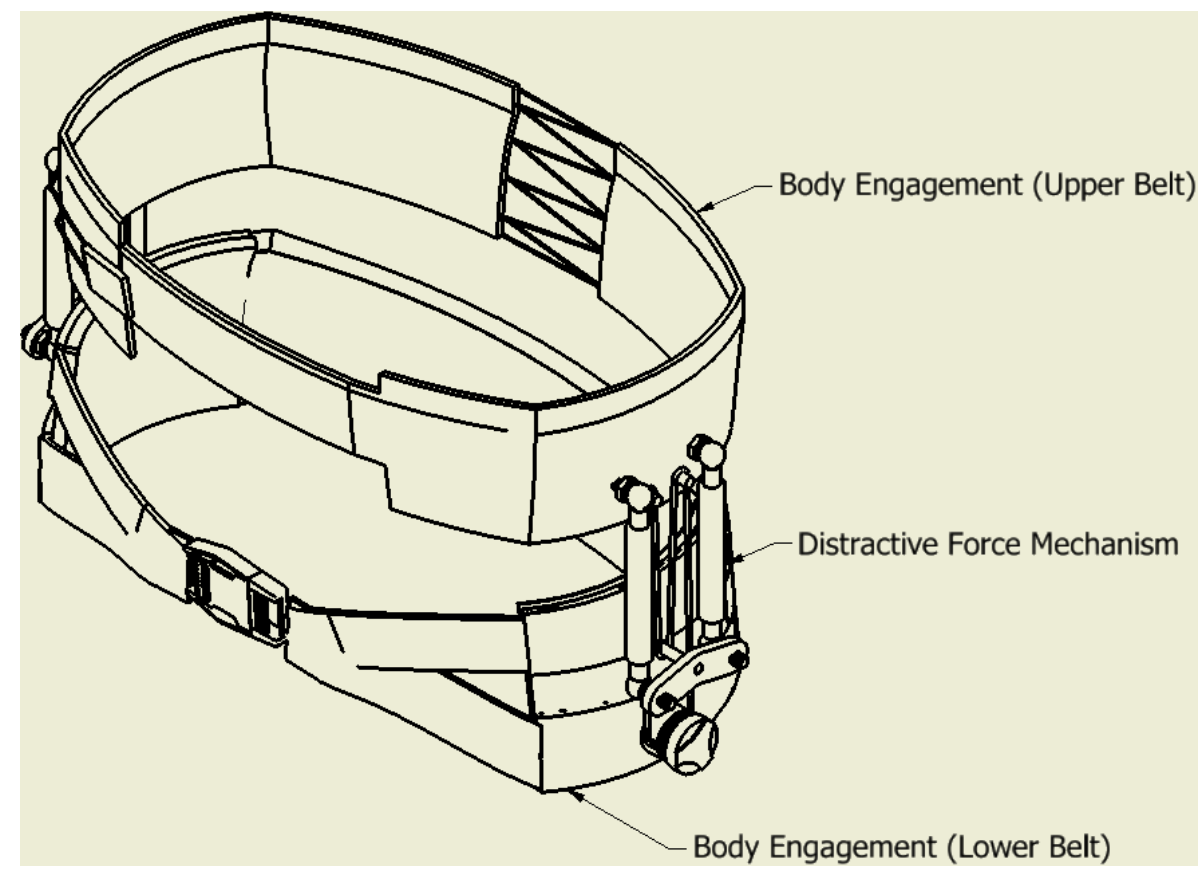

Figure 4-1: DMO4 schematic.

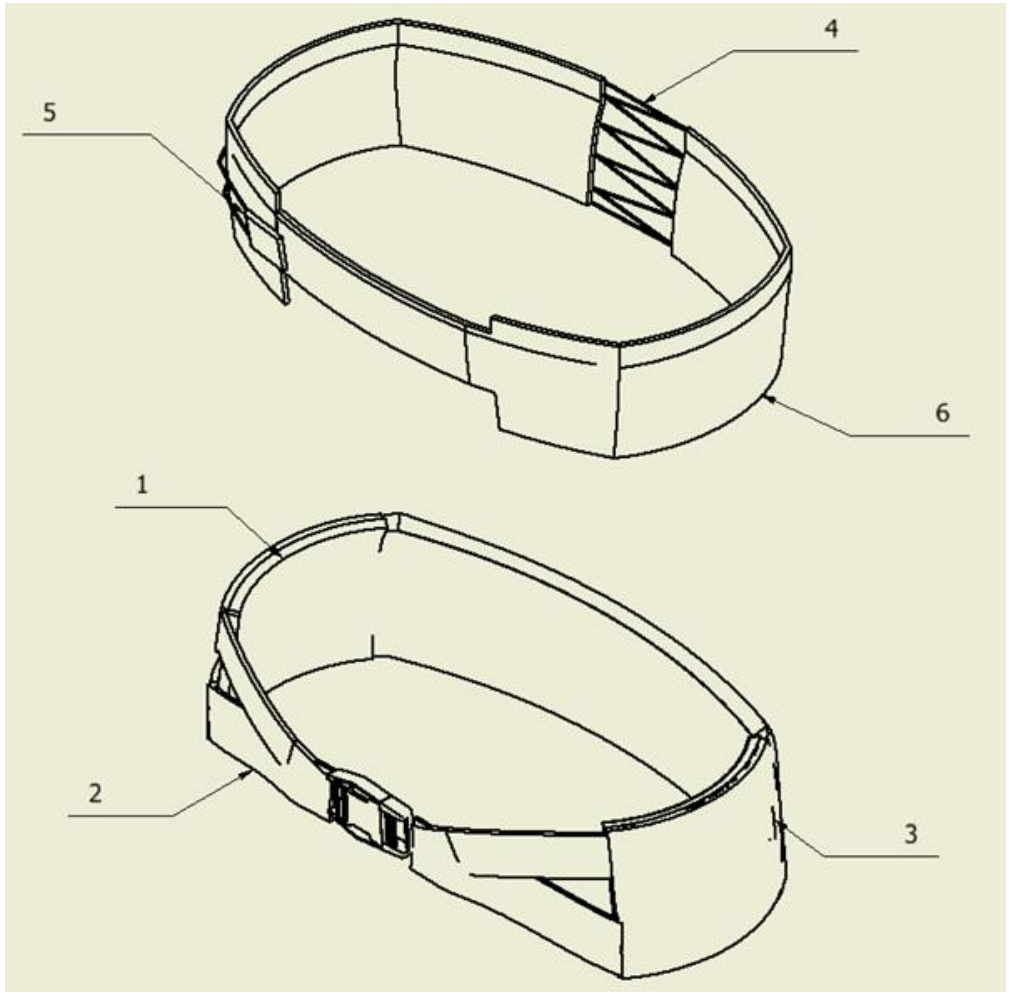

Figure 4-2: Torso and hip belts of DMO4. 
As the distractive force is applied, the DFM interface in the torso and hip belts attempt to separate. As this happens, the body engaging components transfer the force to the patient's hips and torso through conforming pads. Both the hip and torso belts were designed and sent to a soft goods developer so that a small batch of production quality prototypes could be made.

\section{DMO4 Distractive Force Mechanism}

The DFM, shown in Figure 4-3, is the biggest differentiator between DMO3 and DMO4. DMO4 utilizes two pairs of gas springs (7) (American Gas Springs, OR, USA) located on the lateral sides to provide the offloading force. These gas springs have a near constant output much like the constant force springs used in DMO3, but in an off-theshelf package. A tensioning knob (8), located on the hip belt, ratchets down the gas springs with a loop of cable (9) between pulleys (10) located on the torso and hip belt inserts $(11,12)$. When the tension is released, force from the gas springs attempts to separate the two pocket inserts located in the belts. The gas springs are attached with anchored ball joints to the torso and hip belts, and are easily swapped for offloading force adjustments. Each gas spring set can be custom ordered to match desired offloading force. Testing was performed on a set providing $160 \mathrm{~N}$.

\section{DMO4 Mobility-Enabling Component}

The MEC in DMO4 are also depicted in Figure 4-3. The ball joints (13) previously mentioned attach to the top and bottom of the gas springs. These joints are anchored to studs (14) located on the insert within the body engagement belts. The ball joints have unlimited ROM in the sagittal plane and $20^{\circ}$ each in the other two planes. The lower ball joints are fixed to a rocker bar (15) that freely rotates about a pin (16) anchored to the hip belt insert. The pin is located near the anatomical IAR while the belt is worn to prevent unwanted off-axis forces. With this combination of mobility components, DMO4 is able to conform to most any DLA and LSCS exercise. 


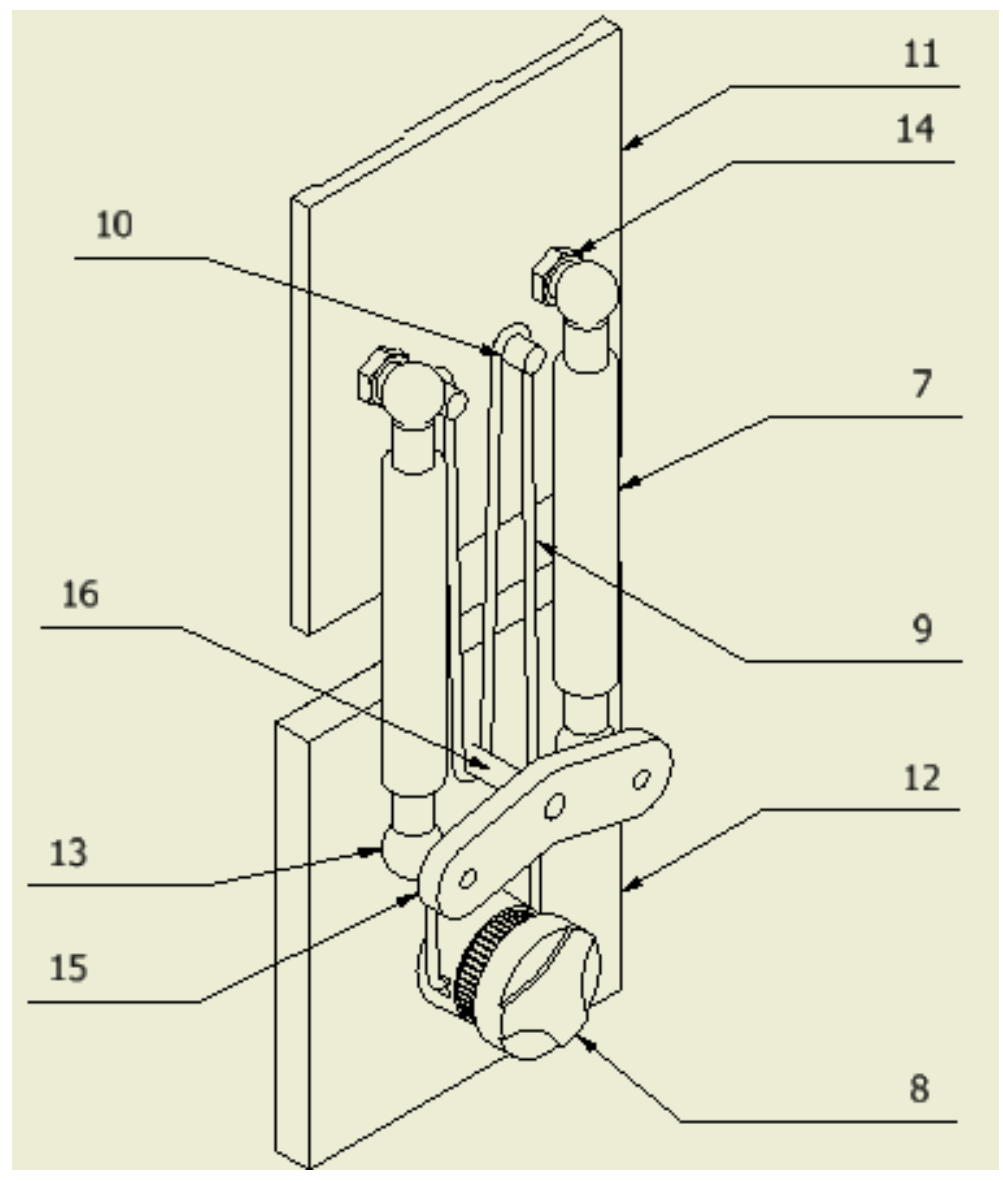

Figure 4-3: DMO4 distractive force mechanism. 


\section{CHAPTER 5. A BIOMECHANICAL EVALUATION OF AN IMPROVED UNLOADING AND MOBILITY-ENABLING LUMBAR SPINAL ORTHOSIS}

With the new synthetic spinal model validated and its IAR mapped, the current version of the back orthosis, DMO4, could be tested on the RTP utilizing similar methods to the previous iterations. Matching the DMO3 performance tests, the RTP placed a 150N vertical load on the spine in vertical stance and through $25^{\circ}$ flexion and $10^{\circ}$ extension. This loading and ROM covered many DLAs and should offload enough weight to simulate a decompressed state. Axial rotation was also tested with DMO3 in the RTP, and a similar test was run with DMO4 to ensure the new DMO4 design changes also provided sufficient axial ROM [21].

\section{Introduction}

Low back pain (LBP) is a prevalent problem by all measures - it affects $30-45 \%$ of the population at any given time [1-5], is one of the top 5 reasons for visits to a healthcare professional $[6,7]$, and is the number one cause of disability for patients under the age of 45 years $[1,8,9]$. A majority of this pain is mechanical in nature [10-13], and initial treatment attempts begin with conservative methods $[11,12]$. This conservative treatment usually begins with over the counter medication followed by many different forms of physical therapy strengthening [7, 14], water therapy, distraction tables, passive therapy (heat packs, ice, TENS), and exercise. When conservative methods do not work, more serious or invasive options such as opioids, spinal injections, or surgery are used to treat LBP.

Unfortunately for some people, pain may be a limiting factor in the success of standard conservative methods. This limited success and the ease of access to narcotics has led to an increase of opioid prescriptions $[6,15,16]$. With the rampant rise of opioid addiction and limitations with many current conservative treatment methods, there remains a need for an alternative conservative approach $[6,17,18]$.

This motivation led to the development of a dynamic Lumbar Sacral Orthosis (LSO) that was designed to apply a distractive load to the lumbar spine while allowing unconstrained movement. This combination of features should decompress the spine and alleviate pain - offering healthcare providers a solution to treat LBP to lessen the occurrence of prescribing addictive opioid medications. The brace functions similarly to a traction table in that it alleviates pain by applying distraction to the spine. Unlike a traction table, however, this orthosis allows for local motion of the lumbar spine in all planes of movement (sagittal, transverse, and coronal) with minimal additional muscle recruitment. The overarching goal of the dynamic LSO was to enable patients to perform standard physical therapy exercises and various DLAs while receiving benefits of traction therapy. 
The previously reported version of the DMO project, DMO2, met the design goal of successfully offloading $300 \mathrm{~N}$ through $25^{\circ}$ of flexion and $10^{\circ}$ of extension [44]. This $300 \mathrm{~N}$ target load enabled the brace to offload a 750N person's upper torso weight [22] through a sagittal plane range suitable for most DLAs [23]. However, DMO2 was not without limitations. DMO2 was a one of a kind prototype that was not easily modifiable, was difficult to properly align, and was limited to sagittal plane motion. Since then, two iterations (DMO3 and DMO4) have been made to improve wearability, simplify the design, and provide configurable options for patients.

The purpose of this testing was to verify that the change in the Distractive Force Component (DFC), Mobility-Enabling Component (MEC), and torso belt does not alter the effectiveness of the brace using previously established testing methods. The design goals for DMO4 were slightly modified from the $300 \mathrm{~N}$ offloading force to a force of $150 \mathrm{~N}$ based on additional testing and experience. DMO4 must be able to offload $150 \mathrm{~N}$ (half of a $750 \mathrm{~N}$ person's upper torso weight) in an upright stance, through $25^{\circ}$ of flexion and $10^{\circ}$ of extension. DMO4 must also allow for $10^{\circ}$ of unconstrained axial rotation, sufficient for most DLAs [23].

\section{Materials}

\section{Distractive and Mobility-Enabling Orthosis}

The current brace (DMO4) (Figure 5-1) can be broken into three main components: the DFC, the MEC, and the body engagement components. The DFC of DMO4 is a set of paired gas springs (American Gas Springs, OR, USA) located on the lateral sides of the brace. These provide a balanced offloading force between the two body engagement components. The MECs featured in this design are the ball joints connecting the DFC and the pinned rotating axis located near L3/L4 on the lower body engagement belt [36]. Body engagement in DMO4 consists of an Osprey Isoform Men's Hipbelt (Osprey Packs, Inc., CO, USA) molded to conform to the patients hips and a back cinching Cybertech SPINE Brace (Compass Health Brands, OH, USA) gripping the patients upper torso. A tensioning knob (Click Medical, CO, USA) is situated on the hip belt that compresses the gas springs prior to application.

This latest design functions similarly to the previous iterations, except for how the forces are applied within the two belts. In DMO2, the user first donned the brace in a zero potential energy state. Then a decompressive force was added by deflecting a pair of rods on the lateral sides of the brace. In DMO4, to eliminate custom machined components and complexity, gas springs replaced the flexible rods and caster as the DFC. With DMO4, the user first compresses the gas springs through the use of the boa tensioning knob, and then releases them once the brace has been put on. An added benefit with this DFC change is that customizable loads can be quickly swapped to match patient needs. A total offloading force of $160 \mathrm{~N}(4 \times 40 \mathrm{~N})$ was selected for the test conditions. 


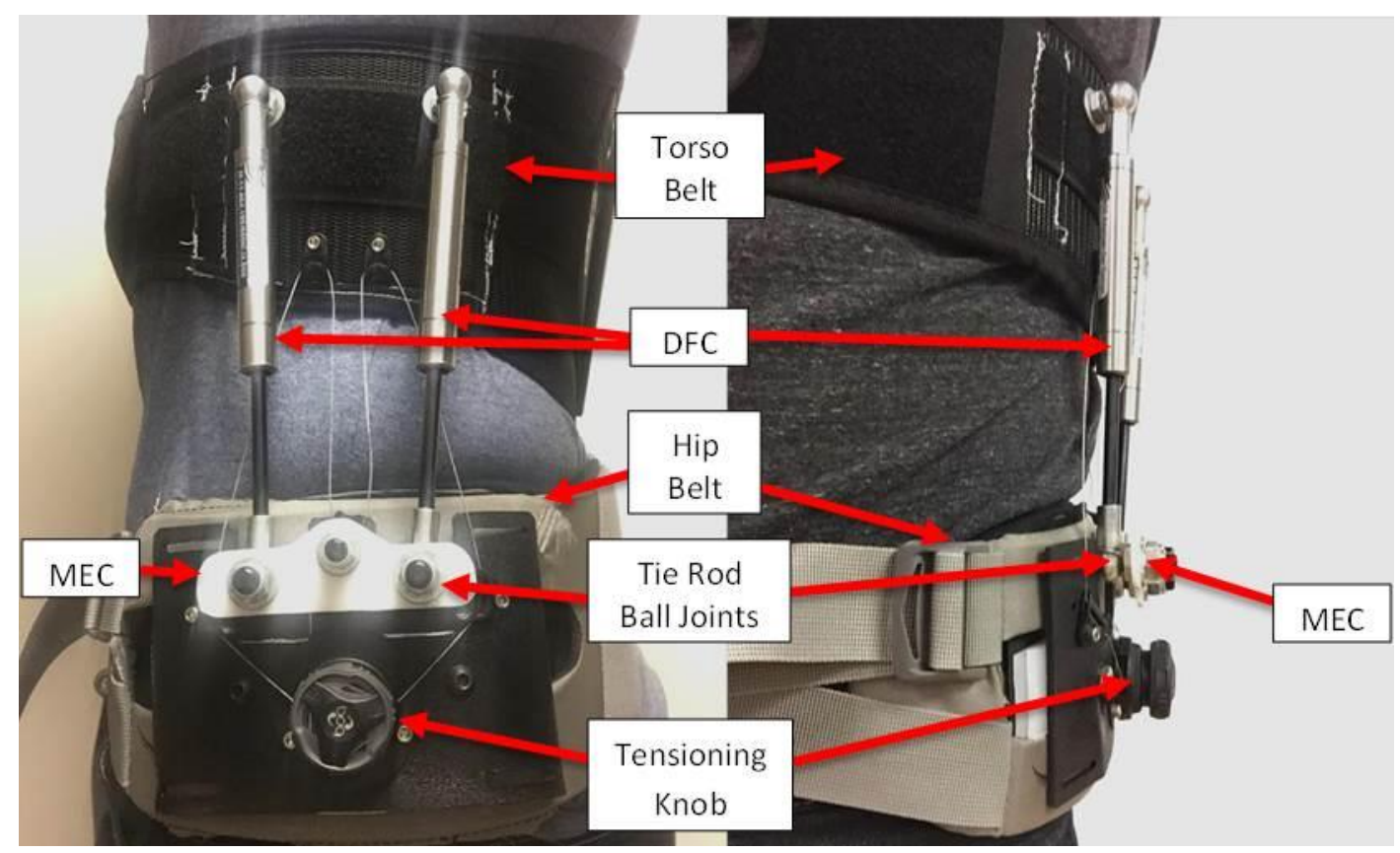

Figure 5-1: Front and side view of DMO4 displaying key components. 
In addition to the change in the DFC, other modifications were made to DMO4. Fixation to the torso and hip belts was integrated between fabric layers using stiff, moldable plastics. This integration removed additional weight and machined parts. The torso belt was swapped with a back cinching belt that more closely conformed to the ribcage. After all changes were made, DMO4 eliminated over 30 parts - both custom machined and stock materials.

\section{Robotic Testing Platform and Analog Model}

Two tests were performed on a multi-axis robotic testing platform (RTP) that was previously used to evaluate the mechanics of existing back braces [44]. The RTP has fine translational movement in 4 axes and a pair of six-axis load cells. One load cell is located on the gimbal assembly and the other mounted to a stationary platform [19]. These are referred to as the Upper Load Cell (ULC) and Bottom Load Cell (BLC), respectively.

A human analog model was previously created from a life-size male mannequin and reinforced in order to withstand the loading conditions necessary for the study [20]. A synthetic lumbar spine, Sawbones \#3430 (Sawbones, WA, USA), was used in place of the previous version after moment/angle and Instantaneous Axis of Rotation (IAR) validation testing [24]. This synthetic spine, in conjunction with the reinforced mannequin, enabled the brace to be tested within the RTP for its offloading capabilities and bending moment required to reach end ranges of motion. Figure 5-2 displays the RTP, mannequin, and synthetic spine.

\section{Methods}

\section{Brace Testing Protocol for Flexion and Extension}

Figure 5-3 depicts the test layout for DMO4 testing. After installing the analog model into the RTP - connecting the two load cells within the frame, DMO4 was aligned and secured first to the pelvic girdle portion located around the bottom load cell and then to the upper torso. Care was taken to ensure that there was no buildup of forces or moments when attaching the analog model to the load cells and that the rotational axis of the brace was in line with the previously determined rotational axis of the synthetic spine. Forces applied via the gimbal assembly are passed through the upper torso and shared between the spine and orthosis. Forces passing through the spine are read at the bottom load cell and forces through the brace are calculated from the difference.

The control strategy for flexion and extension follows a Pure Moment protocol detailed by DiAngelo and Hillyard [67]. To apply the loading condition, the RTP was programmed to establish the kinematic path of the analog model under pure moment application. It accomplishes this task by incrementally rotating about the initial 


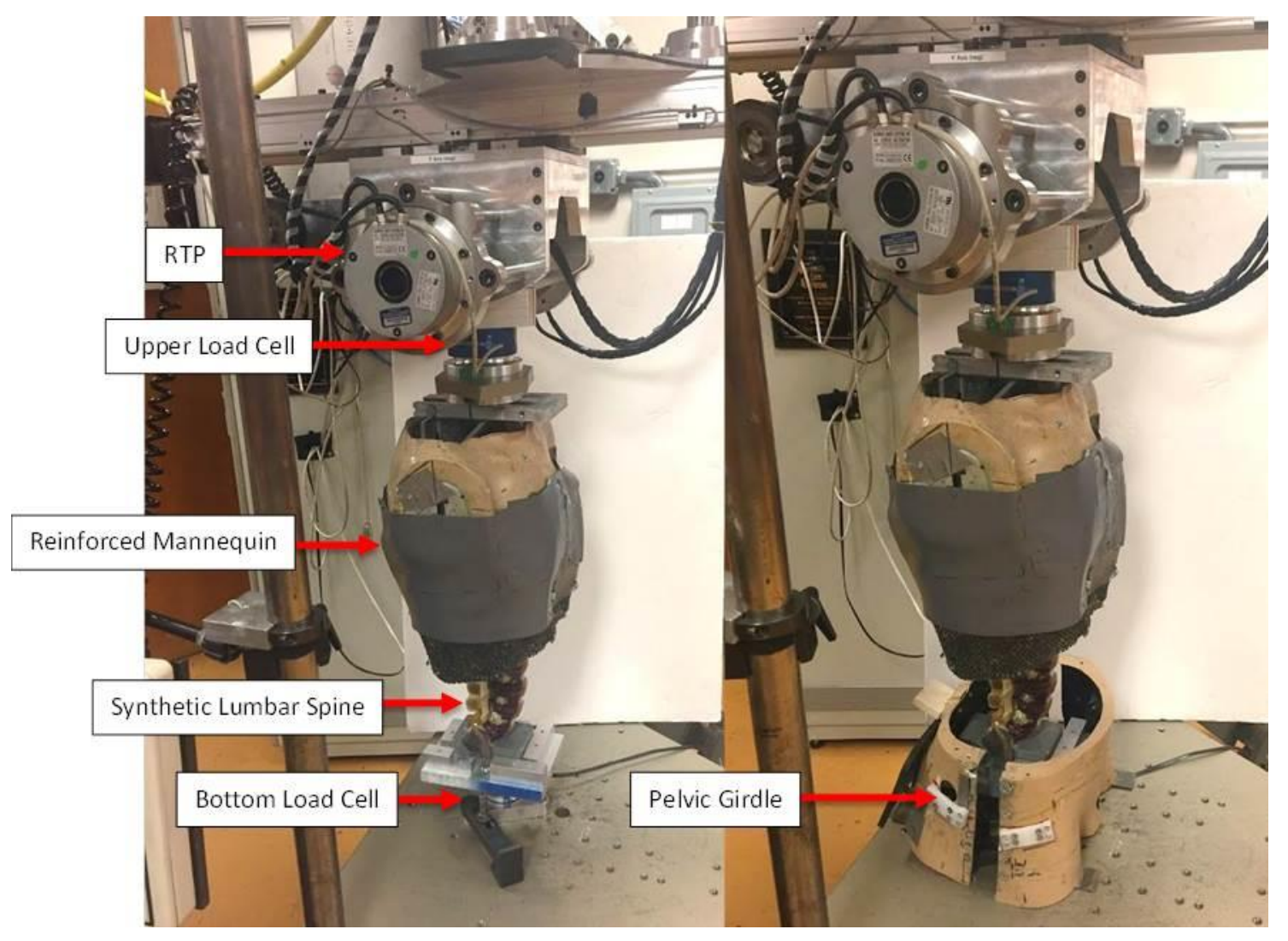

Figure 5-2: RTP and the human analog model with and without the pelvic girdle. 


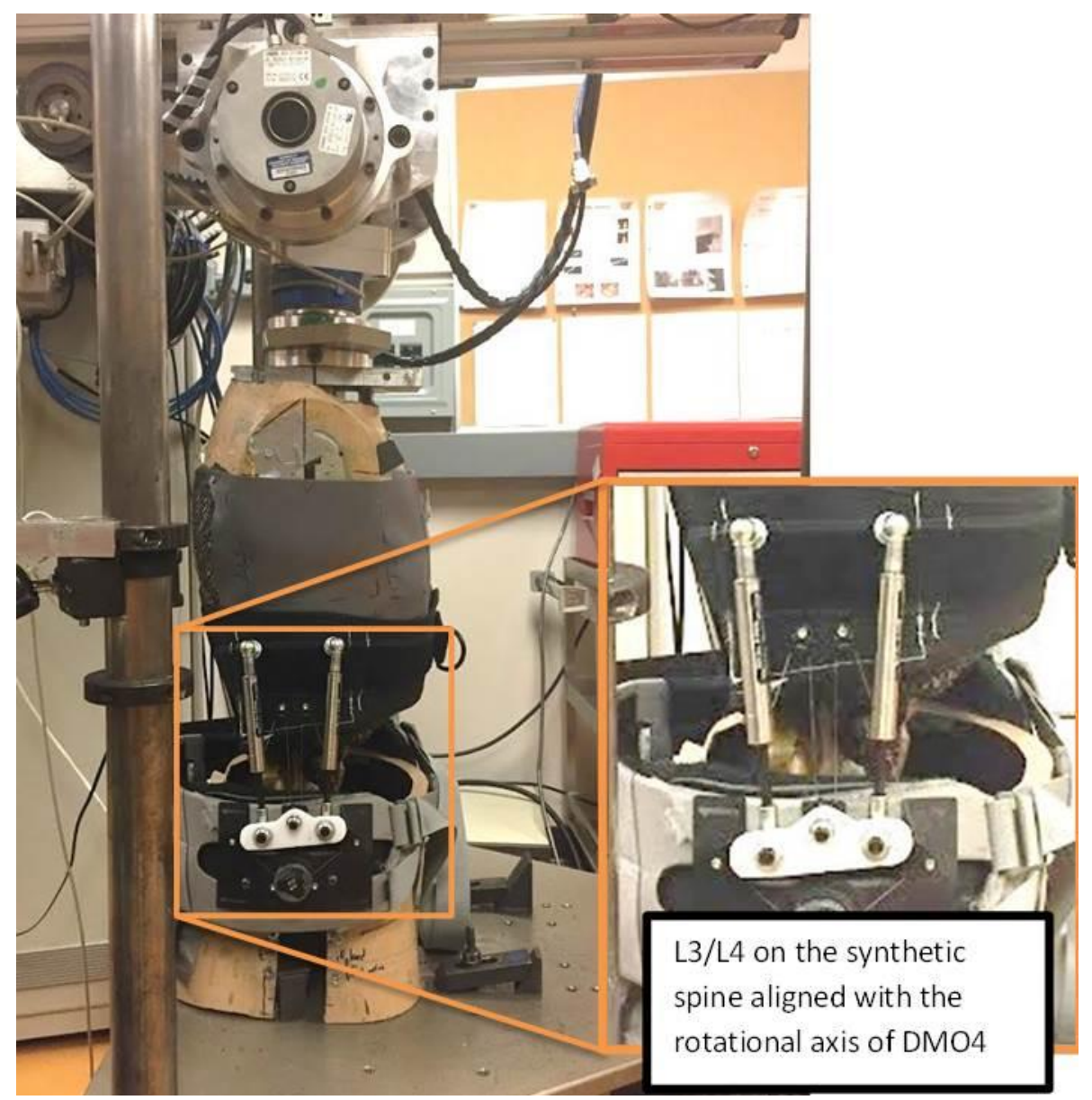

Figure 5-3: DMO4 installed onto the analog model for flexion and extension testing.

The exploded view depicts the rotational axis of DMO4 being aligned with the axis of the lumbar spine. 
programmed point of sagittal rotation and then reducing off-axis forces by translating the gimbal in $\mathrm{x}$ and $\mathrm{z}$ directions. This step minimizes the distance between the initial prescribed Center of Rotation (COR) and the true COR of the lumbar spine. The process was repeated until the desired range of $25^{\circ}$ and $10^{\circ}$ in flexion and extension was met.

\section{Brace Testing Protocol for Axial Rotation}

The axial rotation test was also performed within the RTP with the analog model, but without the Sawbones lumbar spine so that unconstrained motion could be evaluated. Initial setup was the same with the analog model being installed on the upper load cell, but instead of a bottom load cell, a passive turntable was set on the platform and the pelvic girdle mounted to that. The turntable allowed axial rotation between the two human analog halves (Figure 5-4). DMO4 was then mounted to the pelvic girdle and upper torso as before. A digital force scale (Esky Digital Scales, USA) was attached to the turntable to ensure passive rotation did not exceed $0.1 \mathrm{Nm}$ during the test.

With DMO4 installed, the turntable was rotated with the force scale to simulate axial rotation. At each trials end rotation, as determined by the $0.1 \mathrm{Nm}$ limit, the degree of axial rotation was measured with a goniometer.

\section{Data Management}

During flexion and extension testing, applied loads and moments were measured and recorded by the ULC which has an $800 \mathrm{~N}$ load and $40 \mathrm{Nm}$ moment capacity with a $0.4 \mathrm{~N}$ resolution. The ULC measured at a rate of $10 \mathrm{~Hz}$. Transferred loads and moments were measured and recorded by the BLC with a load and moment capacity of $1150 \mathrm{~N}$ and $40 \mathrm{Nm}$. The BLC had a resolution of $0.2 \mathrm{~N}$ and measured at a rate of $15 \mathrm{~Hz}$. Brace load and brace effect were calculated from the difference between the applied and transferred loads and moments.

In the axial rotation protocol, a force scale with a maximum capacity of $489 \mathrm{~N}$ and a resolution of $\pm 0.09 \mathrm{~N}$ was used to pull the brace through its axial rotation range. Read in combination with the set distance from the turntable, the moment applied to rotate the DMO was less than $0.1 \mathrm{Nm}$. At the end limit, the angle between the starting position and end range was recorded from a goniometer. This test was repeated three times to each direction.

\section{Results}

\section{Spinal Off-Loading}

The offloading capacity of the 160N DMO4 prototype is shown in Figure 5-5 for the $150 \mathrm{~N}$ upper body weight loading condition through a range of $25^{\circ}$ flexion and $10^{\circ}$ 


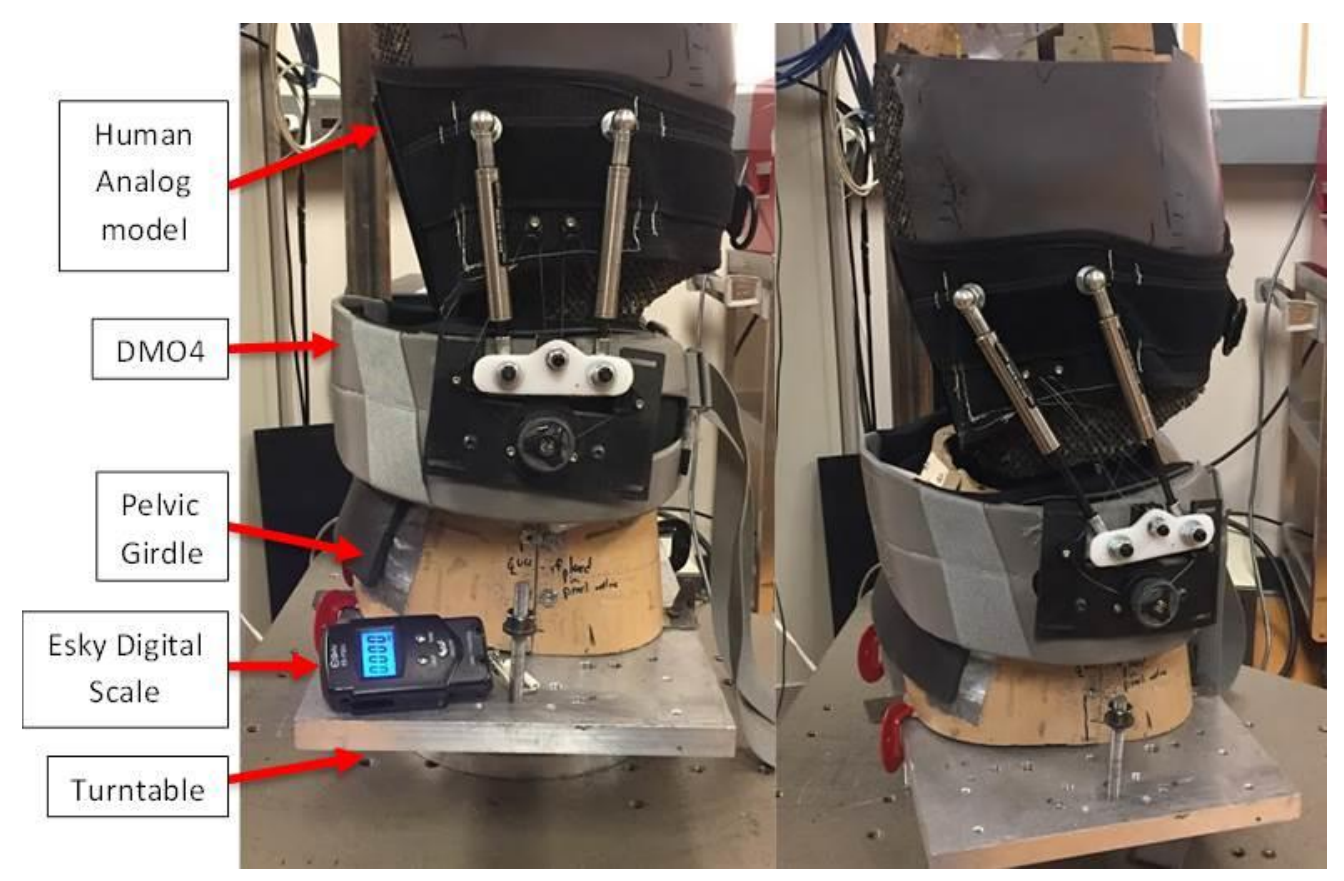

Figure 5-4: Axial rotation test setup.

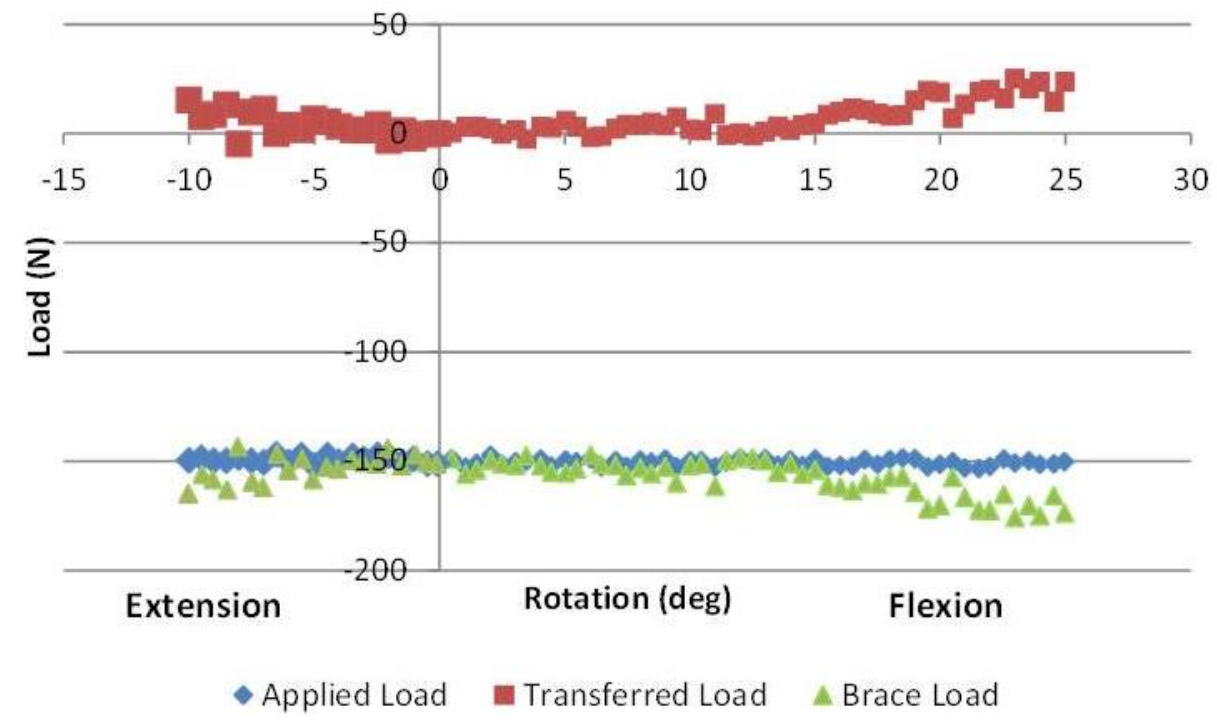

Figure 5-5: Spinal offloading of DMO4 in upright stance and throughout extended ranges of motion.

A positive transferred load represents a net distraction load applied across the spine. 
extension. The percentage of applied load shared with the DMO is provided in Table 5-1 along with loads carried by the DMO and those transferred through the Sawbones spine model. At the flexion end range, DMO4 supported 109\% of the applied loading condition. At the extension end range, DMO4 also supported 109\% of the applied loading condition. Throughout the entire flexion and extension range, DMO4 was able to support the $150 \mathrm{~N}$ load.

\section{Bending Moment}

The bending moment versus angular displacement or brace effect response of DMO4 is shown in Figure 5-6 for the 150N upper body weight loading condition. The brace effect values and the percentage of brace effect to applied and physiological moments are provided in Table 5-2. The brace effect at the flexion end range was $3.2 \mathrm{Nm}$ or $4.8 \%$ of a physiological load [68]. The brace effect at the extension end range was $2.4 \mathrm{Nm}$ or $2.6 \%$ of a physiological load. The brace effect moment of DMO4 is linearly related to the angular displacement.

\section{Axial Rotation}

The results for the three trials in left and right axial twisting can be seen in Table 5-3. For both directions, DMO4 achieved well over $10^{\circ}$ of unconstrained $(<0.1 \mathrm{Nm}$ of resistance) axial rotation measured goniometer.

\section{Discussion}

DMO4 successfully completed the flexion and extension protocol. Loads of $150 \mathrm{~N}$ were fully supported by DMO4 at each incremental degree change and the brace even applied small amounts of distraction at end ranges of motion. This is due to the brace's actual capacity to be near $160 \mathrm{~N}$ (40Nx4 gas springs) causing traction on the spine and lower load cell. Early efficiency losses in DMO4 $(\sim 10 \mathrm{~N})$ may be explained with static friction losses in the gas springs. As the brace was flexed, friction was released and enabled the springs to fully apply their $160 \mathrm{~N}$ force. Brace load compared between DMO3 and DMO4 is shown in Figure 5-7. Steady progress from DMO2 to DMO4 has been made to ensure a consistent offloading force [67]. DMO4 also retained a low brace effect value as seen in the previous models - less than $5 \mathrm{Nm}$ at end ranges of motion. A comparison between DMO3 and DMO4 is shown in Figure 5-8. This resistance is more linear when compared to previous generations and is negligible in the early stages of motion. It is important to keep the brace effect value low, as this affects muscle recruitment and ROM for the patient. $5 \mathrm{Nm}$ is roughly $5 \%$ of the moment from the weight of the upper body in a flexed position [68]. It is also important that the brace be correctly positioned at or near the spine's natural IAR. With previous testing, the Sawbones spine IAR was mapped and the brace could be fitted accordingly [24]. For future use on 
Table 5-1: DMO4 compressive load values at upright stance and at end ranges of motion.

\begin{tabular}{lcccc}
\hline $\begin{array}{c}\text { Degrees of } \\
\text { Rotation }\end{array}$ & $\begin{array}{c}\text { Applied } \\
\text { Load (N) }\end{array}$ & $\begin{array}{c}\text { Transferred } \\
\text { Load (N) }\end{array}$ & $\begin{array}{c}\text { Brace } \\
\text { Load (N) }\end{array}$ & $\begin{array}{c}\text { Brace Load as a } \\
\text { Percentage of } \\
\text { Applied Load (\%) }\end{array}$ \\
\hline At $10^{\circ}$ Extension & 150 & -15 & 164 & 109 \\
At $0^{\circ}$ & 151 & 0 & 151 & 100 \\
At $25^{\circ}$ Flexion & 151 & -14.5 & 165 & 109 \\
\hline
\end{tabular}

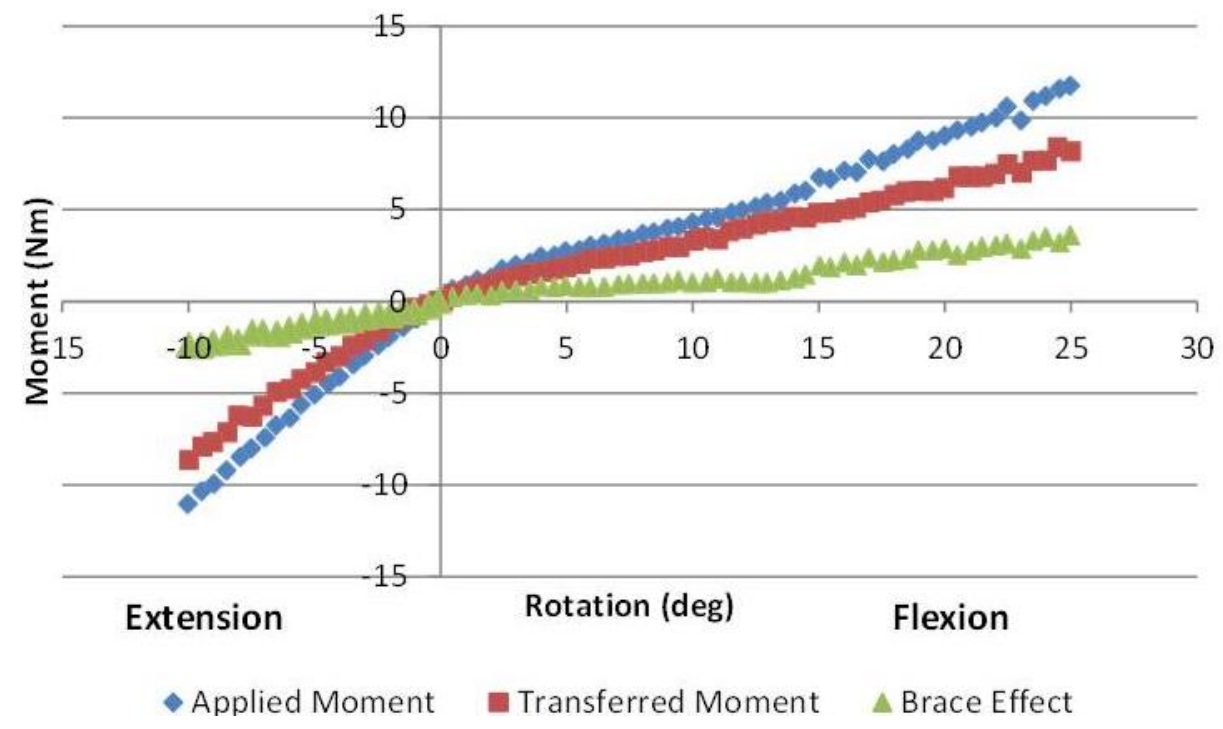

Figure 5-6: Resistance to bending of DMO4 in upright stance and throughout extended ranges of motion. 
Table 5-2: DMO4 bending moment values at end ranges of motion.

\begin{tabular}{lccccc}
\hline $\begin{array}{l}\text { Degrees of } \\
\text { Rotation }\end{array}$ & $\begin{array}{c}\text { Applied } \\
\text { Moment } \\
(\mathbf{N m})\end{array}$ & $\begin{array}{c}\text { Transferred } \\
\text { Moment } \\
(\mathbf{N m})\end{array}$ & $\begin{array}{c}\text { Brace } \\
\text { Effect } \\
\text { (Nm) }\end{array}$ & $\begin{array}{c}\text { Brace Effect } \\
\text { as a \% of } \\
\text { Applied } \\
\text { Moment (\%) }\end{array}$ & $\begin{array}{c}\text { Brace Effect } \\
\text { as a \%of } \\
\text { Physiological } \\
\text { Moment (\%) }\end{array}$ \\
\hline $\begin{array}{l}\text { At } 10^{\circ} \\
\text { Extension }\end{array}$ & 11 & 8.6 & 2.4 & 21 & 2.6 \\
$\begin{array}{l}\text { At } 25^{\circ} \\
\text { Flexion }\end{array}$ & 11.6 & 8.4 & 3.2 & 27 & 4.8 \\
\hline
\end{tabular}

Data Source: M. Nordin and V. Frankel, Basic Biomechanics of the Musculoskeletal System, 2nd ed.: Lea \& Febiger, 1989 [68].

Table 5-3: DMO4's unconstrained $(<0.1 \mathrm{Nm}$ of resistance $)$ axial rotation degree values.

\begin{tabular}{lcccc}
\hline \multicolumn{1}{c}{ Direction of Rotation } & Trial 1 & Trial 2 & Trial 3 & Average \\
\hline Left Axial Twisting (Clockwise) & $30^{\circ}$ & $26^{\circ}$ & $26^{\circ}$ & $27^{\circ}$ \\
Right Axial Twisting (Counterclockwise) & $33^{\circ}$ & $31^{\circ}$ & $33^{\circ}$ & $32^{\circ}$ \\
\hline
\end{tabular}




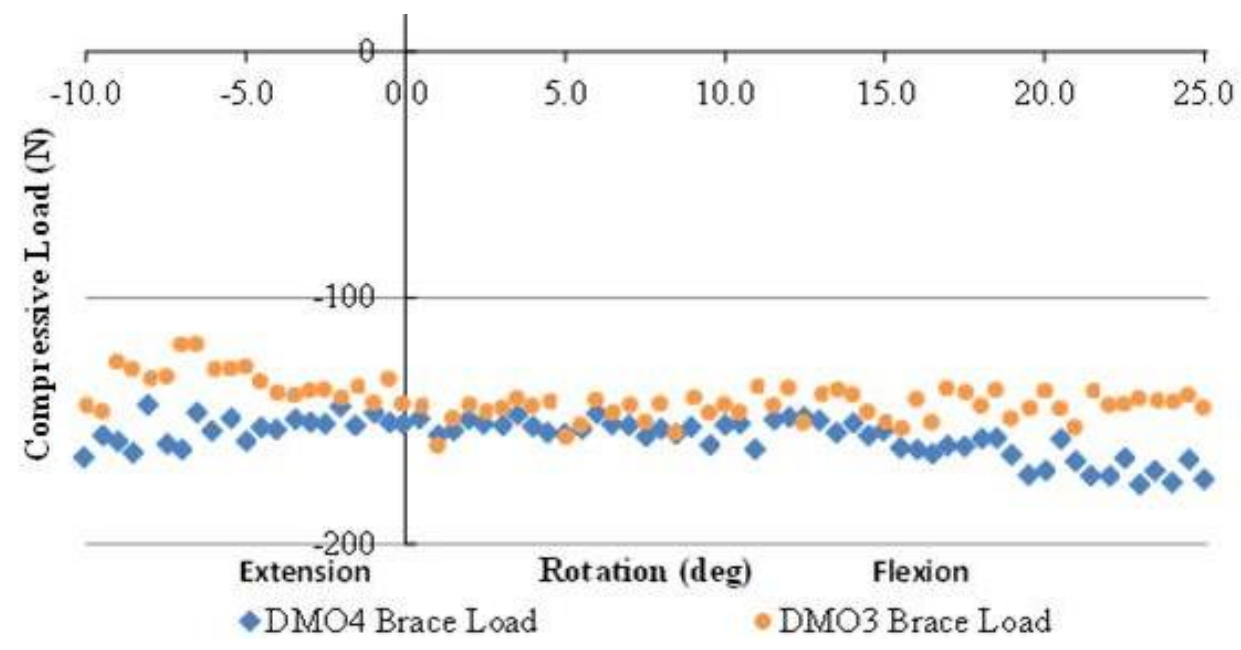

Figure 5-7: Brace offloading capacity or brace load comparison between the latest models.

Data Source: D. C. Hillyard, "A Novel Distractive and Mobility-Enabling Lumbar Spinal Orthosis for Treating Mechanical Low Back Pain," Theses and Dissertations (ETD), 2017 [21].

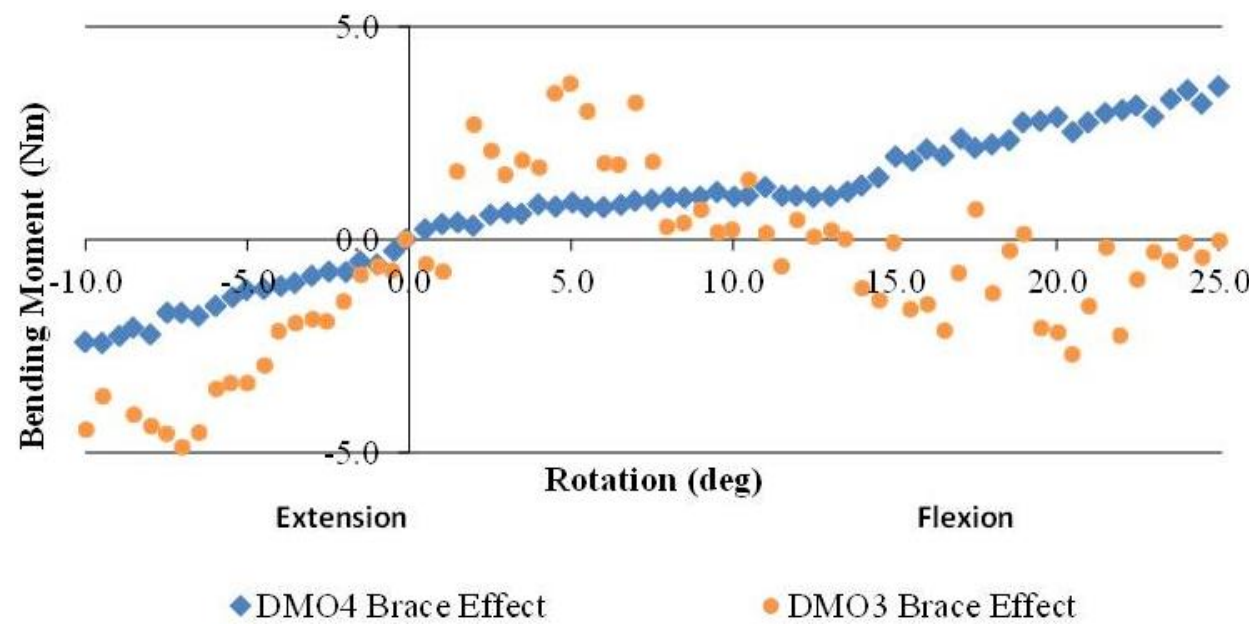

Figure 5-8: Resistance to bending or brace effect comparison between the latest models.

Data Source: D. C. Hillyard, "A Novel Distractive and Mobility-Enabling Lumbar Spinal Orthosis for Treating Mechanical Low Back Pain," Theses and Dissertations (ETD), 2017 [21]. 
patients, the initial location of the brace will be based on the lumbar spine rotating near the middle of the L3/L4 intervertebral disc[36].

During the axial rotation test, $\mathrm{DMO} 4$ reached an average of almost $30^{\circ}$ in both directions, more than triple the range required for most DLAs [23]. Adding unconstrained axial rotation was a big step from DMO2 to DMO3 and DMO4. The DFC of DMO4 is connected to the braces through the use of ball joints that allow for $10^{\circ}$ of rotation in each direction, more than enough to handle DLAs. Combined with the natural twisting of the DFC and the gas spring's ability to shorten and lengthen as necessary, DMO4 is able to allow the patient to undergo a ROM necessary to complete exercises and daily tasks while providing sufficient offloading.

The limitations with this study were similar to past brace testing studies involving the RTP. The RTP can simulate the force components of the gravitational load on a human torso, but not the in vivo spinal bending moment. The Sawbones spine was designed to imitate an average healthy person and not a patient with mechanical LBP. Permanent alteration of the synthetic spine would be necessary to simulate pathology associated with LBP. Motion during the flexion and extension tests were locked to the sagittal plane and no coupled loading conditions were simulated. Even with these limitations, the range for most activities was able to be simulated and the RTP was successful in validating the capacity of DMO4 throughout the designed ROM.

\section{Conclusion}

Like DMO2, the newly revised DMO4 successfully offloaded the target capacity with minimal brace effect - less than 5Nm over the entire ROM in the sagittal plane. This biomechanical equivalency coupled with the ability to axially rotate and the reduction in complexity, number of components, and profile of the torso belt yielded the next generation in the dynamic off-loading LSO project. 


\section{CHAPTER 6. DISCUSSION}

\section{Additional Synthetic Spine Tests - Eccentric Load Condition}

With regards to the lumbar spine model, the published paper documents the testing on the RTP as only using a PM protocol. Similarly to the DMO PM protocol requiring a seeded initial rotation point, the PM testing with the synthetic spine model required one as well. To find this seed location, an eccentric load (EL) protocol was used. Test setup is shown in Figure 6-1. The rod and pivoting bearing are the key to this protocol - as the bearing is displaced vertically, the rod forces the spine to bend and slips in or out depending on the direction [36]. This action does not bias the spine to rotate about any specific point. Markers at each level track the spine's natural motion. Using Crisco's technique [54] for tracking bodies in space, a global IAR for the spine is created from the markers at T12, shown in Figure 6-2. The initial rotation point for future PM protocols began at the L3L4 disc space since most of the IAR points lie within that area and is backed by previous studies [36].

\section{DMO4 Comparison to DMO3}

\section{Design Changes Between DMO3 and DMO4}

In the latest DMO iteration, DMO4, the hip belt and design concepts from DMO3 largely remain the same. Steps were taken to simplify the design, eliminate custom components, and remove unwanted profile from under the arm (Figure 6-3). The torso belt was swapped for a belt mimicking a cinching Cybertech SPINE brace which allowed for a stronger grip on the upper torso and was more conforming to the body. The tensioning knob was moved to the hip belt, away from the patient's elbow and the DFM to belt interface was integrated into the belt fabric. The main difference between DMO3 and DMO4 is the DFM. DMO4 utilizes readily available gas springs as opposed to the custom machined components found in DMO3. These changes resulted in fewer parts, an under-arm profile reduction of 1.5 ", and a brace that could easily change the force output by swapping the gas springs.

\section{DMO3 and DMO4 Robotic Testing Results}

Both DMO3 and DMO4 successfully offloaded 150N in upright stance, $25^{\circ}$ flexion, and $10^{\circ}$ extension. They were both also successful in axial rotation - close to $10^{\circ}$ in DMO3 and $30^{\circ}$ in DMO4. DMO4 steadily improved on the brace effect moment during the sagittal bending tests. DMO4's response was linear and negligible throughout the ROM, maxing out below 5 Nm at the end ranges. DMO3's brace effect was also below $5 \mathrm{Nm}$ throughout its range, but it did not follow a linear pattern and was prone to variation throughout its range. 


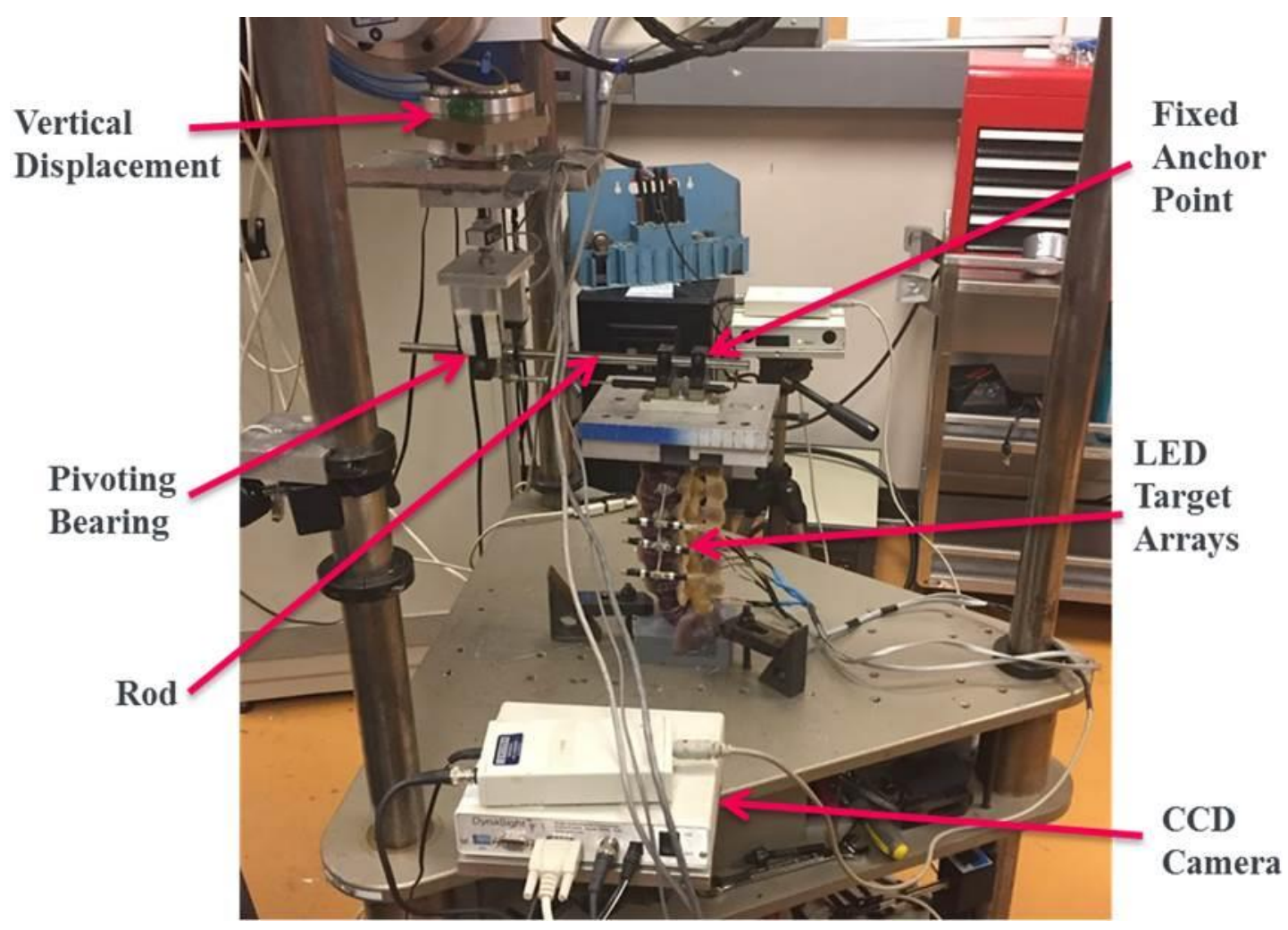

Figure 6-1: $\quad$ Eccentric load test setup.

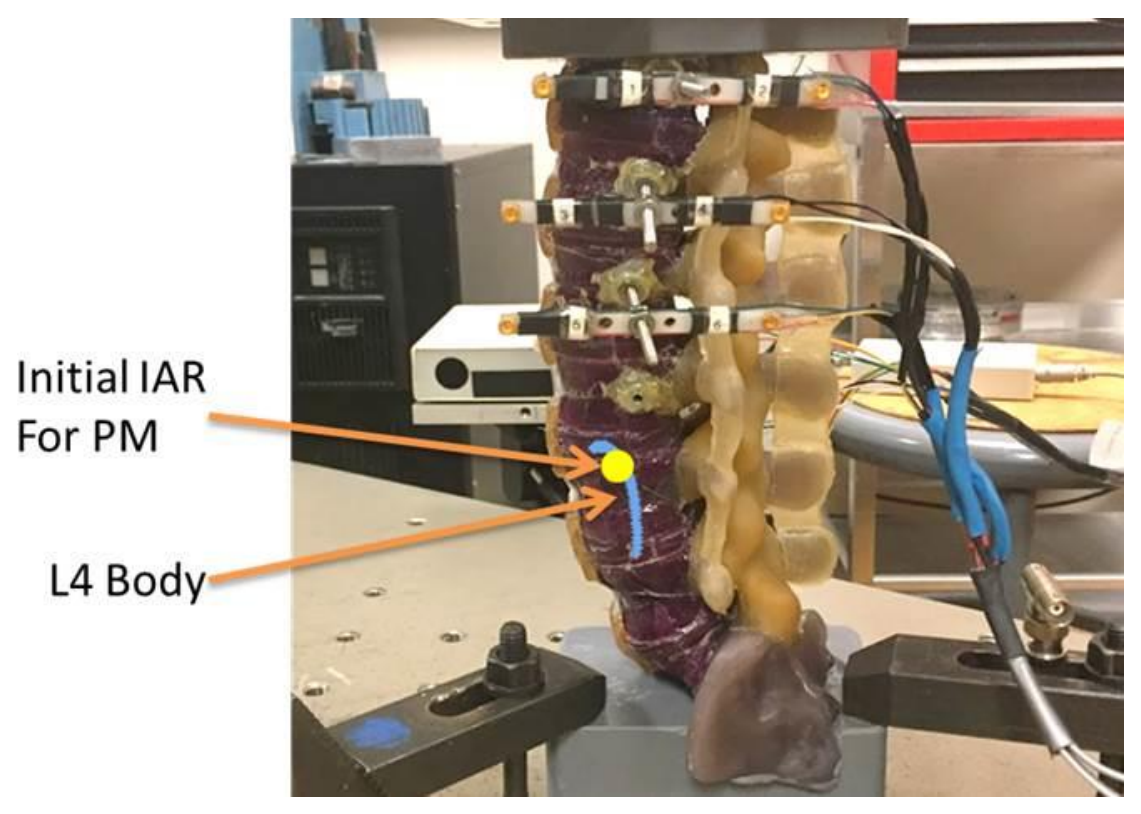

Figure 6-2: Global IAR found from EL protocol and initial rotation for PM protocol. 


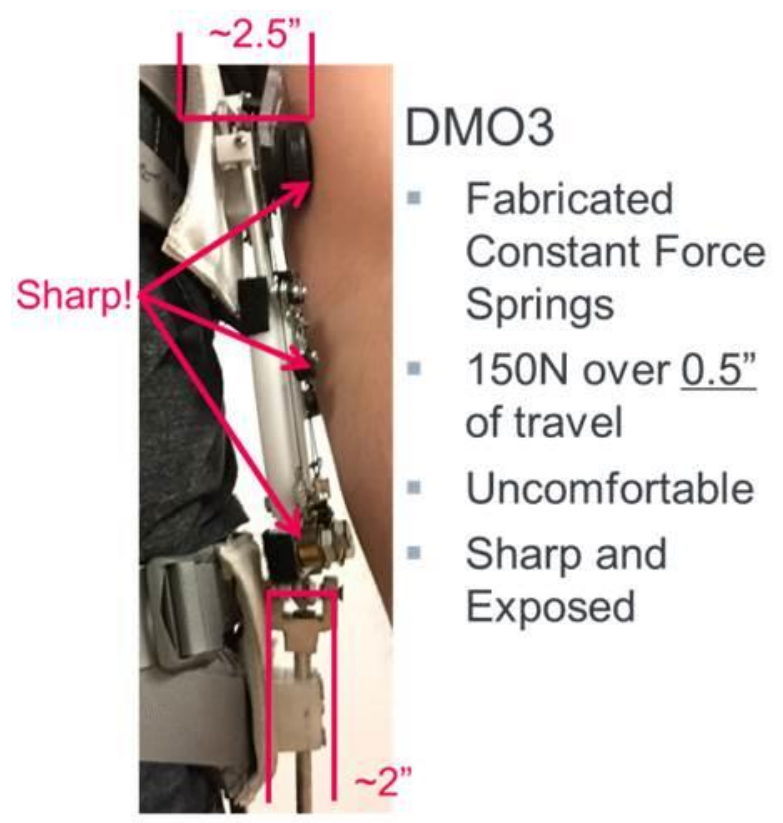

Previous Brace: DMO3

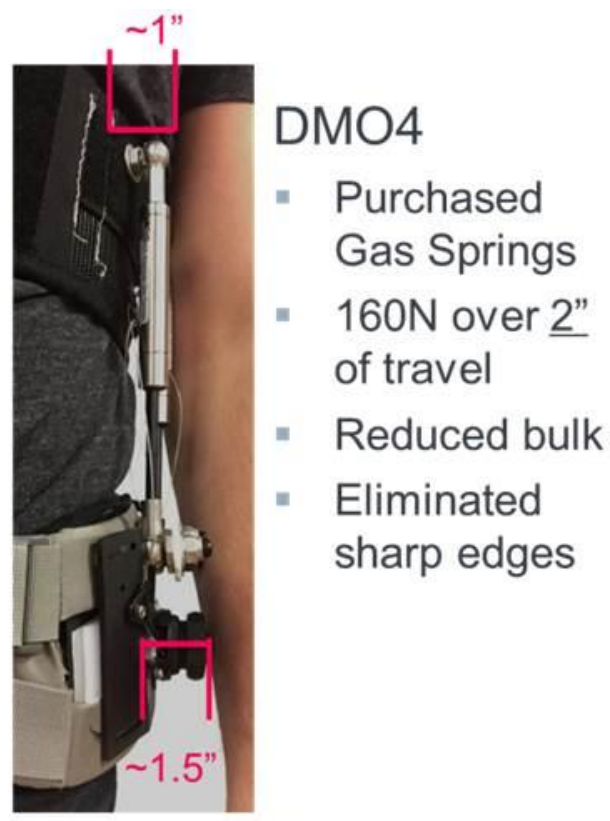

Current Brace: DMO4

Figure 6-3: $\quad$ Profile view comparison of DMO3 and DMO4. 


\section{Patient Case Study of DMO4}

DMO4 was eligible to be pilot tested in the clinic under the previous DMO IRB protocol: \#16-04546-FB. In this pilot, Dr. Richard Kasser, PhD, a physical therapist for University Therapists on the UTHSC campus enrolled a patient with mechanical LBP to wear the brace for six therapy sessions.

The objectives for the clinical study were to determine DMO4's immediate and short-term effects on mechanical LBP and DMO4's effect on quality of life. To test these objectives, two types of scores were collected from the patient. The first was the Numerical Pain Rating Score (NPRS) which is an 11-point verbal pain scale with increased pain at higher numbers. The second was a modified Oswestry Disability Index (mODI), a 10 question survey designed to quantify how much pain affects a patient's ability to perform DLAs. Outcomes for this patient were based on the NPRS and mODI scores. At each visit, a 30\% decrease or 2-point drop in NPRS score was said to be clinically significant (Minimal Clinically Important Difference) [69]. The mODI scores from before the first session with the DMO to last session were compared. A mODI difference was clinically significant if there was a change of 10 percentage points [69, 70].

\section{Clinical Testing for Pilot Patient}

This male patient had previously attended therapy sessions, but was unsuccessful in lowering pain to a satisfactory level. At the start of the trial sessions, the patient completed a mODI after signing the informed consent documents. A NPRS score was taken at the start and end of each therapy session in which the patient performed LSCS exercises as directed by Dr. Kasser. The exercises utilized included prone leg raises, trunk lifts, planks, crunches, and treadmill walking. The six sessions lasted an average of 27 minutes over a 4 week period. At the conclusion of six sessions, a second mODI score and overall satisfaction score was recorded.

\section{Results}

This pilot patient completed six therapy sessions with DMO4. Results for the pain scores before and after wearing DMO4 at each therapy visit and mODI scores before and after the round of therapy can be seen in Figure 6-4 and Figure 6-5, respectively. Dotted lines in the figures represent clinically significant differences in both pain and mODI scores. For this patient, DMO4 provided a clinically meaningful decrease in pain, down to a score of 1, after each therapy session. DMO4 also lowered the mODI score for the pilot patient to $16 \%$ from $24 \%$, nearing clinical significance and transitioning the patient from moderate disability to minimal disability according to the interpretation of the mODI scores [71]. 


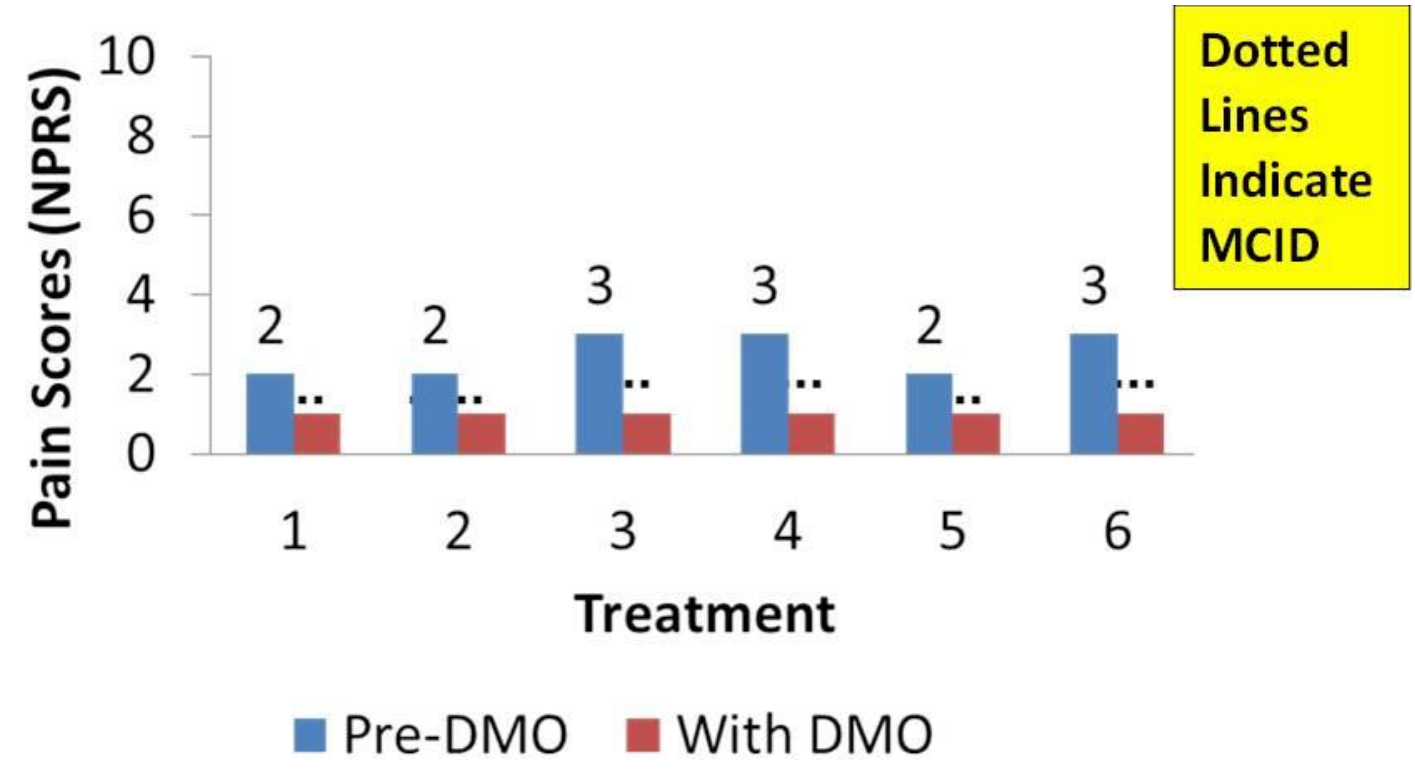

Figure 6-4: NPRS scores for patient 1 before and after wearing DMO4 during PT visits.

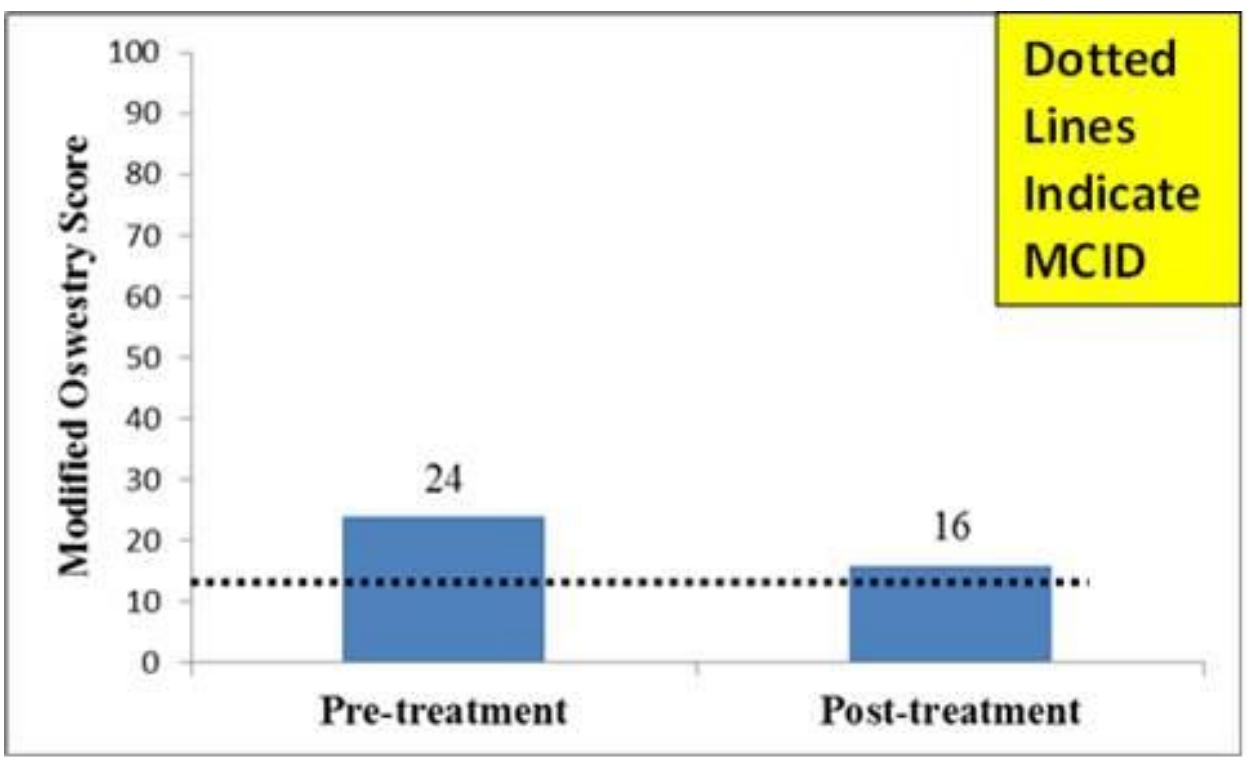

Figure 6-5: mODI scores for patient 1. 


\section{Discussion}

This patient was relatively healthy with generally low pain and mODI scores. Nonetheless, he still had LBP that flared up and caused issue for daily work and home life activities. Patient 1 completed the six in-brace therapy sessions and was successful in lowering his pain to a score of 1 after each session and dropped a category in mODI scores. With the patient having previously attended therapy sessions without DMO4, gathering these results from the sessions with the DMO yielded a success for the pilot study.

\section{DorsaVi $^{\mathrm{TM}}$ Clinical Application}

Another potential clinical study aspect with the DMO project is utilizing DorsaVi sensors to track patient outcomes. DorsaVi with Low Back Module is a clinical tool that includes a set of sensors placed on the lumbar spine of a patient and is used to track ROM, EMG of paraspinal muscles, and lumbar orientation. These results can then be compared to approved reference data that matches the patient's demographics and the patient's past results. Utilized within the clinical study with the DMO, these types of results could further test DMO4's low brace effect values in flexion and extension by reporting EMG values. DorsaVi could also be used to test a patient's ROM with and without the DMO over a series of therapy sessions.

Sample results for flexion are shown in Figure 6-6. This data set is of a healthy subject in DMO4 with a flexion lumbar ROM equal to $39^{\circ}\left(42^{\circ}\right.$ total sagittal ROM), well above the necessary limit for most DLAs [23]. At the top of the figure, patient data sets are shown to the left and the healthy reference values at the right. Similarly, the movement pattern with EMG for three flexion movements is shown near the bottom. The alert for overactive EMG also occurred in the no brace condition and, as such, is likely due to the subject/DorsaVi interface and not the DMO. 

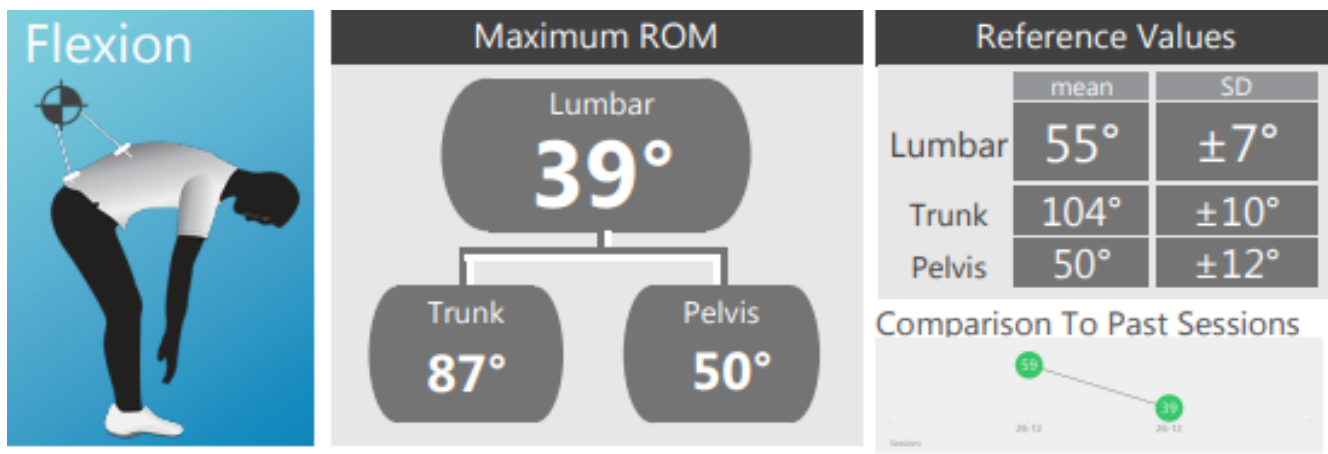

Pain Alert Recorded Comparison

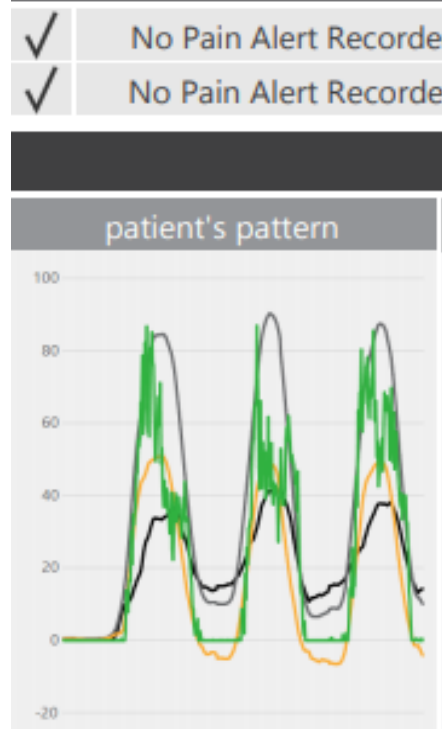

\section{Current Previous}

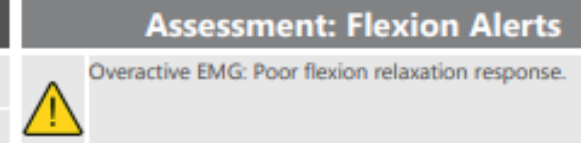

\section{Movement Pattern}

reference data

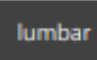

Lumbar is the difference between the trunk and pelvis sensor angles, indicating lumbar movement.

120

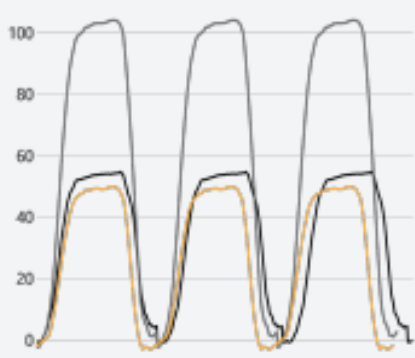

trunk

Trunk indicates the angle of the upper sensor.

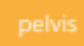

Pelvis indicates the angle of the lower sensor. the erector spinae muscles.

Figure 6-6: DorsaVi ${ }^{\mathrm{TM}}$ low back module flexion results of a healthy patient wearing DMO4.

This image was generated using ViMove ${ }^{\circledR}$ software (DorsaVi, NY, USA) in Dr. DiAngelo's lab on 10/13/2018 using research data. 


\section{CHAPTER 7. CONCLUSION}

The prototype biomimetic spine utilized in the original testing of DMO1, DMO2 and DMO3 was replaced with a more robust model. This synthetic lumbar spine model has been validated and integrated into the testing procedure. The synthetic lumbar spine model had a repeatable and known IAR path, and closely resembled the biomechanical properties of human cadaver spines without creating a biohazard environment. This update to the testing protocol aided in the design verification of the latest DMO model and enabled easy comparison between model updates and future competitor braces. The latest orthosis, DMO4, maintained the ability to offload the spine throughout a range of tested motion and reduced complex parts so as to simplify the manufacturability of the brace. DMO4 also had a low bending moment, requiring minimal effort from the patient, and thus relying on the use of the patient's own muscle involvement to carry our various DLAs. The laboratory testing provided a unique method that confirmed design

functionality goals were met while the orthosis was simplified. Pilot clinical data yielded positive results for the dynamic LSO project's latest iteration and will hopefully lead to more clinical testing opportunities in the future. 


\section{LIST OF REFERENCES}

[1] R. A. Deyo, "Conservative Therapy for Low Back Pain: Distinguishing Useful From Useless Therapy," JAMA, vol. 250, pp. 1057-1062, 1983.

[2] J. Hartvigsen, M. J. Hancock, A. Kongsted, Q. Louw, M. L. Ferreira, S. Genevay, et al., "What low back pain is and why we need to pay attention," The Lancet, vol. 391, pp. 2356-2367, 2018.

[3] G. Andersson, "Epidemiological features of chronic low-back pain," The Lancet, vol. 354, pp. 581-585, 1999.

[4] A. D. Woolf and B. Pfleger, "Burden of major musculoskeletal conditions," Bulletin of the World Health Organization, vol. 81, pp. 646-656, 2003.

[5] F. Balague, A. F. Mannion, F. Pellise, and C. Cedraschi, "Non-specific low back pain," Lancet, vol. 379, pp. 482-91, Feb 42012.

[6] R. A. Deyo, M. Von Korff, and D. Duhrkoop, "Opioids for low back pain," Bmj, vol. 350, p. g6380, Jan 52015.

[7] J. O. Tavee and K. H. Levin, Low Back Pain vol. 23, 2017.

[8] T. Vos, C. Allen, M. Arora, R. M. Barber, Z. A. Bhutta, A. Brown, et al., "Global, regional, and national incidence, prevalence, and years lived with disability for 310 diseases and injuries, 1990\&2013;2015: a systematic analysis for the Global Burden of Disease Study 2015," The Lancet, vol. 388, pp. 1545-1602, 2016.

[9] S. Bigos, O. Bowyer, G. Braen, and K. Brown, "Acute low back problems in adults: assessment and treatment. Agency for Health Care Policy and Research," Clin Pract Guidel Quick Ref Guide Clin, pp. iii-iv, 1-25, Dec 1994.

[10] J. S. Will, D. C. Bury, and J. A. Miller, "Mechanical Low Back Pain," Am Fam Physician, vol. 98, pp. 421-428, Oct 12018.

[11] "Back Pain Fact Sheet," NINDS, 2014.

[12] Chronic Back Pain. Available: https://my.clevelandclinic.org/health/diseases/16869-chronic-back-pain

[13] S. Eidelson, MD. (2019). 6 Causes of Mechanical Back Pain. Available: https://www.spineuniverse.com/conditions/back-pain/6-causes-mechanical-backpain

[14] E. A. Shipton, "Physical Therapy Approaches in the Treatment of Low Back Pain," Pain and therapy, vol. 7, pp. 127-137, 2018.

[15] T. J. Hudson, M. J. Edlund, D. E. Steffick, S. P. Tripathi, and M. D. Sullivan, "Epidemiology of regular prescribed opioid use: results from a national, population-based survey," Journal of pain and symptom management, vol. 36, pp. 280-288, 2008.

[16] W. M. Compton and N. D. Volkow, "Major increases in opioid analgesic abuse in the United States: concerns and strategies," Drug Alcohol Depend, vol. 81, pp. 103-7, Feb 12006.

[17] M. D. Sullivan and C. Q. Howe, "Opioid therapy for chronic pain in the United States: promises and perils," Pain, vol. 154 Suppl 1, pp. S94-S100, 2013.

[18] J. E. Couto, M. C. Romney, H. L. Leider, S. Sharma, and N. I. Goldfarb, "High rates of inappropriate drug use in the chronic pain population," Popul Health Manag, vol. 12, pp. 185-90, Aug 2009. 
[19] B. P. Kelly and D. J. DiAngelo, "A Multiaxis Programmable Robot for the Study of Multibody Spine Biomechanics Using a Real-Time Trajectory Path Modification Force and Displacement Control Strategy," Journal of Medical Devices, vol. 7, pp. 034502-034502-7, 2013.

[20] J. C. Simmons, "Development of a Mobility-Enabling Spinal Orthosis and Methods for Evaluating and Developing Spinal Orthoses on a Robotic Platform," Theses and Dissertations (ETD), 2014.

[21] D. C. Hillyard, "A Novel Distractive and Mobility-Enabling Lumbar Spinal Orthosis for Treating Mechanical Low Back Pain," Theses and Dissertations (ETD), 2017.

[22] D. Winter, Biomechanics of Human Movement. New York: Wiley, 1979.

[23] J. E. Bible, D. Biswas, C. P. Miller, P. G. Whang, and J. N. Grauer, "Normal functional range of motion of the lumbar spine during 15 activities of daily living," J Spinal Disord Tech, vol. 23, pp. 106-12, Apr 2010.

[24] D. J. Diangelo, Hoyer, D., Chung, C., "Biomechanical evaluation of a full-length (T12-S) synthetic lumbar spine model," MOJ Applied Bionics and Biomechanics, vol. 3, 2019.

[25] D. Synthes. Anatomy of the Spine. Available: https://www.depuysynthes.com/patients/aabp/recognizingsymptoms/anatomyofth espine

[26] M. Adams, The Biomechanics of Back Pain vol. 1. Edinburgh, Scotland: Elsevier Science Ltd, 2002.

[27] J. G. Betts, K. A. Young, J. A. Wise, E. Johnson, B. Poe, D. H. Kruse, et al., Anatomy \& Physiology. pp. 264: OpenStax 2013, https://openstax.org/books/anatomy-and-physiology/pages/7-3-the-vertebralcolumn

[28] D. Hoy, L. March, P. Brooks, F. Blyth, A. Woolf, C. Bain, et al., "The global burden of low back pain: estimates from the Global Burden of Disease 2010 study," Ann Rheum Dis, vol. 73, pp. 968-74, Jun 2014.

[29] M. Modic, "Lumbar Degenerative Disk Disease," Radiology, vol. 245, pp. 43-61, 2007.

[30] D. J. Gonzalez-Blohm SA, Lee WE, Shea TM, Aghayev K, Vrionis FD, "The current testing protocols for biomechanical evaluation of lumbar spinal implants in laboratory setting: A Review of the literature," Biomedical Research International, pp. 1-15, 2015.

[31] V. K. Goel, et al., "Biomechanical testing of the spine. Load-controlled versus displacement-controlled anaylsis," Spine, vol. 20(21), pp. 2354-7, 1995.

[32] M. M. Panjabi, et al., "Mechanical behavior of the human lumbar and lumbosacral spine as shown by three-dimensional load-displacement curves," The Journal of Bone and Joint Surgery, vol. 76(3), pp. 413-24, 1994.

[33] A. G. Patwardhan, et al., "A follower load increases the load-carrying capacity of the lumbar spine in compression," Spine, vol. 24(10), pp. 1003-9, 1999.

[34] M. M. Panjabi, "Hybrid multidirectional test method to evaluate spinal adjacent level effects," Clinical Biomechanics, vol. 22(3), pp. 257-65, 2007. 
[35] B. Kelly, "A Multiaxis programmable spine robot for the study of multibody spinal biomechanics using real-time hybrid force and displacement control strategies," Doctoral Dissertation, The University of Tennessee Health Science Center, 2005.

[36] J. R. Stubbs, "Use of a multi-axis robotic testing platform to investigate the sagittal mechanics of the multi-body lumbar spine," Thesis and Dissertations (ETD), The University of Tennessee Health Science Center, 2011.

[37] J. Campbell, Imsdahl, S, Ching, RP, "Evaluation of a synthetic functional spine unit," in Northwest Biomechanics Symposium, 2011.

[38] J. Domann, Mar, D, Johnson, A, Johnson, A, James, J, Friis, E, "The analogue spine model: The first anatomically and mechanically correct synthetic physical model of the lumbar spine," The Spine Journal, vol. 11(10), pp. S155-S156.

[39] J. Domann, "Development and validation of an analogue lumbar spine model and its integral components," Master's Thesis, University of Kansas, 2011.

[40] T. Wang, Ball, JR, Pelletier, MH, Walsh, WR, "Initial experience with synthetic spinal motion segments: biomechanical assessment of high cycle and implant performance," ed. Poster Presentation: Orthopedic Research Society Meeting, 2014.

[41] J. Campbell, Imsdahl, S, Ching, RP, "Evaluation of a Synthetic L2-L5 Spine model for biomechanical testing," ed. Poster Presentation: Canadian Biomechanics Society, 2012.

[42] W. Camisa, Leasure, JM, Buckley, JM, "Biomechanical validation of a synthetic lumbar spine," ed. Poster Presentation: 2014 Annual Meeting of the North American Spine Society, 2014.

[43] T. Wang, Ball, JR, Pelletier, MH, Walsh, WR, "Biomechanical evaluation of a biomimetic spinal construct," Journal of Experimental Orthopedics, vol. 1:3, 2014.

[44] D. DiAngelo, Hillyard, DC, "A novel distractive and mobility-enabling lumbar spinal orthosis," Journal of Rehabilitation and Assistive Technologies

Engineering, vol. 3, pp. 1-10, 2016.

[45] J. Cossette, Farfan, HF, Robertson, GH, Wells, RV, "The instantaneous center of rotation of the third lumbar intervertebral joint," J Biomech, vol. 4(2), pp. 149-53, 1971.

[46] S. Gertzbein, Holtby, R, Tile, M, Kapasouri, A, Chan, KW, Cruickshank, B, "Determination of a locus of instantaneous centers of rotation of the lumbar disc by moiré fringes. A new technique.," Spine, pp. 409-13, 1984.

[47] T. Haher, O’Brien, M, Felmly, WT, et al., "Instantaneous axis of rotation as a function of three columns of the spine," Spine, vol. 17 (6S), pp. S149-54, 1992.

[48] V. Haughton, Rogers, B, Meyerand, ME, Resnick, DK, "Measuring the axial rotation of lumbar vertebrae in vivo with MR imaging," AJNR Am J Neuroradiol, vol. 23(7), pp. 1110-6, 2002.

[49] M. Inoue, Mizuno, T, Sakakibara, T, Kato, T, Yoshikawa, T, Inaba, T, Kasai, Y, "Trajectory of instantaneous axis of rotation in fixed lumbar psine with instrumentation," J Orthop Surg Res, 2017.

[50] M. Pearcy, Bogduk, N, "Instantaneous axes of rotation of the lumbar intervertebral joints," Spine, vol. 13(9), pp. 1033-41, 1988. 
[51] T. Sakamaki, Katoh, S, Sairyo, K., "Normal and spondylolytic pediatric spine movements with reference to instantaneous axis of rotation.," Spine, pp. 141-5, 2002.

[52] D. Sengupta, Demetropoulos, CK, Herkowitz, HN, "Instant axis of rotation of L45 motion segment - a biomechanical study on cadaver lumbar spine," J Indian Med Assoc., vol. 109(6), p. 389, 2011.

[53] Q. Xia, Wang, S, Kozanek, M, Passias, P, Wood, K, Li, G, "In-vivo motion characteristics of lumbar vertebrae in sagittal and transverse planes," Journal of Biomechanics, vol. 43, pp. 1905-1909, 2010.

[54] J. Crisco, Chen, X, Panjabi, MM, Wolfe, SW, "Optimal marker placement for calculating the instananeous center of rotation," J Biomech, vol. 27(9), pp. 11837, 1994.

[55] B. Kelly, Zufelt, NA, Sander, EJ, DiAngelo, DJ, "The influence of fixed sagittal plane centers of rotation on motion segment mechanics and range of motion in the cervical spine," J Biomech, vol. 46(7), pp. 1369-75, 2013.

[56] D. DiAngelo, Foley, KT, Morrow, BP, Wong, P, Kiehm, K, Sin, A, Bertagnoli, R, Abjornson, A, Kelly, BP, "In vitro testing of lumbar disc arthroplasty devices," The Open Spine Journal, vol. 6, pp. 9-25, 2014.

[57] K. Salb, Wido, DM, Stewart, TE, DiAngelo, DJ, "Development of a robotic assembly for analyzing the instantaneous axis of rotation of the foot ankle complex," Applied Bionics and Biomechanics, pp. 1-9, 2016.

[58] A. Ahmadi, Maroufi, N, Behtash, H, Zekavat, H, Parnianpour, M, "Kinematic analysis of dynamic lumbar motion in patients with lumbar segmental instability using digital videofluoroscopy," Eur Spine J., pp. 1677-85, 2009.

[59] J. Dvorak, Panjabi, MM, Chang, DG, Theiler, R, Grob, D., "Functional radiographic diagnosis of the lumbar spine. Flexion-extension and lateral bending," Spine, pp. 562-71, 1991.

[60] M. Hayes, Howard, TC, Gruel, CR, Kopta, JA, "Roentgenographic evaluation of lumbar spine flexion-extension in asymptomatic individuals," Spine, vol. 14(3), pp. 327-31, 1989.

[61] S. Lee, Wong, KW, Chan, MK, Yeung, HM, Chiu, JL, Leong, JC, "Development and validation of a new technique for assessing lumbar spine motion," Spine, vol. 27(8), pp. 215-20, 2002.

[62] M. Pearcy, Portek, I, Shepherd, J, "Three-dimensional x-ray analysis of normal movement in the lumbar spine. ," Spine, vol. 9(3), pp. 294-7, 1984.

[63] A. White, Panjabi, MM, Clinical biomechanics of the spine, 1990.

[64] I. Yamamoto, et al., "Three-dimensional movements of the whole lumbar spine and lumbosacral joint," Spine, vol. 14(11), 1989.

[65] P. Bifulco, et al., "A continuous description of intervertebral motion by means of spline interpolation of kinematic data extracted by videofluoroscopy," J Biomech, vol. 45(4), pp. 634-41, 2012.

[66] Z. Liu, et al, "Sagittal plane rotation center of lower lumbar spine during a dynamic weight-lifting activity.," J Biomech, vol. 49(3), pp. 371-5, 2016.

[67] D. J. DiAngelo and D. C. Hillyard, "A novel distractive and mobility-enabling lumbar spinal orthosis," RATE, vol. 3, pp. 1-10, 2016. 
[68] M. Nordin and V. Frankel, Basic Biomechanics of the Musculoskeletal System, 2nd ed.: Lea \& Febiger, 1989.

[69] O. Hägg, "The clinical importance of changes in outcome scores after treatment for chronic low back pain," Eur Spine J, vol. 12, pp. 12-20, 2003.

[70] R. W. Ostelo and H. C. de Vet, "Clinically important outcomes in low back pain," Best Pract Res Clin Rheumatol, vol. 19, pp. 593-607, Aug 2005.

[71] J. Fairbank, J. Couper, J. Davies, and J. O'Brien, "The Oswestry low back pain disability questionnaire," Physiotherapy, vol. 66, pp. 271-273, 1980. 


\section{VITA}

Daniel Hoyer was born to Keith and Cheryl Hoyer of Brookport, Illinois in 1992. He graduated from Massac County High School in May of 2010. He then went on to study mechanical engineering at Harding University and graduated in May 2014. Soon after, he moved with his wife to Memphis, Tennessee. After two years of working as a CAD/design engineer at Trinity Meyer Utility Structures, he had the opportunity to return to school and complete a master's degree in biomedical engineering. Under the tutelage of Dr. DiAngelo, he furthered research on a lumbar brace and collected data on a number of studies involving bracing technology within the lab. Daniel expects to receive his Master of Science degree in December 2019. 